\title{
1 TGF-beta/Activin ligand Myoglianin couples muscle growth to the initiation of
}

2 metamorphosis

3

4

5 Lorrie L. He ${ }^{1 \#}$, Sara Hyun Joo Shin ${ }^{1 \#}$, Zhou Wang ${ }^{1}$, Isabelle Yuan ${ }^{1}$, Ruthie Weschler ${ }^{1}$, Allie

6 Chiou, Takashi Koyama ${ }^{2,3}$, H. Frederik Nijhout ${ }^{4}$, Yuichiro Suzuki ${ }^{1 *}$

$13{ }^{1}$ Department of Biological Sciences, 106 Central St., Wellesley College, Wellesley, MA 02481

$14{ }^{2}$ Instituto Gulbenkian de Ciência, Rua da Quinta Grande, 6, 2780-156 Oeiras, Portugal

$15{ }^{3}$ Department of Biology, University of Copenhagen, Universitetsparken 15, 2100 Copenhagen, 16 Denmark

$17{ }^{4}$ Department of Biology, Duke University, Durham, NC 27708

18

19 \# denotes equal contribution

20 *Corresponding author: email: ysuzuki@wellesley.edu; Tel: (781)283-3100

21

22

23

24

25

26 


\section{ABSTRACT}

29 Although the mechanisms that control growth are now well understood, the mechanism by which

30 animals assess their body size remains one of the great puzzles in biology. The final larval instar

31 of holometabolous insects, after which growth stops and metamorphosis begins, is specified by a

32 threshold size. We investigated the mechanism of threshold size assessment in the tobacco

33 hornworm, Manduca sexta. The threshold size was found to change depending on the amount of

34 exposure to poor nutrient conditions whereas hypoxia treatment consistently led to a lower

35 threshold size. Under these various conditions, the mass of the muscles plus integuments was

36 correlated with the threshold size. Furthermore, the expression of myoglianin (myo) increased at

37 the threshold size in both M. sexta and Tribolium castaneum. Knockdown of myo in T.

38 castaneum led to larvae that underwent supernumerary larval molts and stayed in the larval stage

39 permanently even after passing the threshold size. We propose that increasing levels of Myo

40 produced by the growing tissues allow larvae to assess their body size and trigger metamorphosis

41 at the threshold size.

\section{Keywords}

46 Myoglianin; threshold size; body size; muscle growth; Manduca sexta 


\section{BACKGROUND}

In animals that undergo determinate growth, the juvenile stage, during which growth occurs, is separated from the adult stage. The two stages are often separated by a major developmental transition, such as puberty or metamorphosis, which involves physiological, morphological and behavioral changes. Because these developmental transitions occur once the juveniles have grown to a particular body size, organisms must have evolved mechanisms to assess their body size. Although the molecular, genetic and physiological mechanisms that control growth, puberty and metamorphosis are now well-known, the mechanism by which animals assess their body size and determine when to stop growing remains a major unresolved issue in developmental biology.

Holometabolous insects, insects that undergo complete metamorphosis, exhibit determinate growth. These insects grow by undergoing several molts. At the end of the last larval stage, they stop feeding and growing, and metamorphose into the pupal and adult stages that do not grow. The size a larva attains during the last larval instar therefore defines the size of the adult insect. Previous studies have shown that the decision to stop growing and begin metamorphosis is marked by the attainment of a precise body size called the threshold size (Nijhout, 1975).

The threshold size was first identified in the tobacco hornworm, Manduca sexta (Nijhout, 1975). M. sexta typically undergo five larval instars in laboratory conditions. However, when $M$. sexta larvae are fed a low-nutrient diet, larvae grow more slowly and can undergo 1, 2 or 3 additional instars before entering metamorphosis. The threshold checkpoint occurs at the beginning of each instar (Kingsolver, 2007; Nijhout, 1975). A larva below the threshold size at the beginning of an instar will undergo additional molts and increase its body size. Once a larva is above the threshold size when it molts, it enters the last larval instar and will metamorphose at

71 the end of that instar (Kingsolver, 2007; Nijhout, 1975).

Artificial selection on body size has demonstrated that threshold size can evolve,

73 indicating that there is a genetic component to the mechanism that determines the threshold size

74 (Grunert et al., 2015). There are two equivalent measures that can detect threshold size: It can be 75 measured as the width of the head capsule, or as the mass of a larva at the beginning of the instar 76 (Grunert et al., 2015; Nijhout, 1975). Both measures are equivalent. Thus, some mechanism of

77 size sensing must exist that is somehow associated with these measures of body size. 
Recent studies have identified several molecular regulators whose disruption causes supernumerary molts or precocious metamorphosis. These molecular regulators affect the production of, or sensitivity to, juvenile hormone $(\mathrm{JH})$. $\mathrm{JH}$ modifies the actions of the molting hormone, 20-hydroxyecdysone, to prevent the organism from progressing from one life history stage to the next (Riddiford, 1996). JH acts by binding to the basic helix-loop-helix-Per-Arnt-

83 Sim domain protein receptor, Methoprene-tolerant (Met) (Konopova and Jindra, 2007). In flour

84 beetle Tribolium castaneum, knockdown of Met causes larvae to have reduced sensitivity to $\mathrm{JH}$ 85 and undergo early metamorphosis, forming miniature adults (Konopova and Jindra, 2007).

86 Silencing the expression of the JH-response gene, Krüppel homolog $(K r-h 1)$, also leads to

87 precocious metamorphosis in this species (Minakuchi et al., 2009).

It has long been known that removing the corpora allata, the glands that secrete $\mathrm{JH}$, can cause premature metamorphosis, resulting in a dwarf adult. JH-deficient larvae of holometabolous insects, including M. sexta, T. castaneum and the silkworm Bombyx mori, undergo precocious metamorphosis (Daimon et al., 2012; Minakuchi et al., 2008; Ohtaki et al., 1971; Suzuki et al., 2013; Tan et al., 2005). In T. castaneum, knockdown of ventral veins lacking $(v v l)$ leads to precocious metamorphosis by suppressing the production of $\mathrm{JH}$ (Cheng et al., 2014). Recently, the ecdysone response gene, E93, has been shown to be necessary to terminate $\mathrm{JH}$ secretion in order to initiate the onset of metamorphosis; knockdown of E93 leads to the induction of supernumerary molts in T. castaneum (Chafino et al., 2019). Chafino et al (2019) demonstrated that in this species, starvation before day 1 of the final instar can induce supernumerary molts whereas starvation after day 1 does not lead to supernumerary molts. Chafino et al (2019) suggested that the mass on the first day of the final instar likely corresponds to the threshold size and demonstrated that it is associated with an increase in E93 expression

102 from that used in M. sexta and the identified stage may be more consistent with the attainment of 103 irreversible pupal commitment, it is clear that the decision to become a pupa (and hence the 104 threshold size) is already reached $24 \mathrm{hrs}$ after the molt to the final instar. Although the clearance 105 of $\mathrm{JH}$ is a prerequisite for the decision to metamorphose, removal of JH is likely only to be the 106 proximate mechanism that allows larvae to initiate metamorphosis and not part of the mechanism 107 by which a larva assesses its size. 
Hemimetabolous insects are characterized by incomplete metamorphosis, in which

109

110

111

112

113

114

115

116

117

118

119

120

121

122

123

124

125

126

127

128

129

130

131

132

133

134

135

136

137

138 nymphs metamorphose directly into adults. To our knowledge, no study has demonstrated the existence of a threshold size in this group of insects. In these insects, JH also plays a role in the timing of adult development. Knockdown of $K r-h 1$ in the penultimate nymphal instar of the firebug, Pyrrhocoris apterus, the German cockroach, Blattella germanica, and the brown planthopper, Nilaparvata lugens, leads to precocious adult development (Konopova et al., 2011; Li et al., 2018; Lozano and Belles, 2011). In addition, silencing myoglianin (myo), a gene coding for one of the ligands of the TGF-beta/Activin signaling pathway, has been shown to cause extra nymphal molts (Ishimaru et al., 2016; Kamsoi and Belles, 2019): Increased expression of myo has been shown to trigger the final nymphal instar in Blattella germanica (Kamsoi and Belles, 2019). Myo expression in the corpora allata/corpora cardiaca in the fifth (penultimate nymphal) instar nymphs leads to the repression of the JH biosynthesis gene, jhamt, in the sixth (last) instar nymphs (Kamsoi and Belles, 2019). Likewise, in the field cricket, Gryllus bimaculatus, RNA interference (RNAi)-mediated knockdown of myo leads to supernumerary molts accompanied by an increase in jhamt expression (Ishimaru et al., 2016). These studies demonstrate that Myo regulates $\mathrm{JH}$ production in hemimetabolous insects. In addition, Myo has been implicated in ecdysteroid production as the expression of the ecdysone biosynthesis gene neverland decreases in response to myo RNAi (Kamsoi and Belles, 2019).

The role of myo in holometabolous insects is not well known except for its functions in muscles (Augustin et al., 2017; Lo and Frasch, 1999). Myo shares a 46\% amino acid sequence identity with the vertebrate Bone Morphogenetic Protein 11 (BMP11 or Growth differentiation factor 11 (GDF11)) and Myostatin (or GDF8) (Lo and Frasch, 1999). In vertebrates, Myostatin was first isolated in mice and characterized for its role in halting skeletal muscle growth (McPherron et al., 1997; Whittemore et al., 2003). In Drosophila, Myo is expressed in embryonic muscles as well as glial cells (Lo and Frasch, 1999). During the larval stage, Myo acts like Myostatin at the neuromuscular junction (NMJ) to suppress synaptic transmissions and NMJ growth and branching (Augustin et al., 2017). In addition, knockdown of muscle-derived myo leads to increased muscle size and overall body size, indicating that Myo inhibits muscle growth (Augustin et al., 2017). However, myo mutant Drosophila larvae do not undergo extra larval molts (Augustin et al., 2017). In fact, unlike most other insects, the final instar Drosophila larvae do not respond readily to $\mathrm{JH}$; topical application of $\mathrm{JH}$ fails to dramatically delay metamorphosis 
139

140

141

142

143

144

145

146

147

148

149

150

151

152

153

154

155

156

157

158

159

160

161

162

163

and does not induce supernumerary molts (Riddiford and Ashburner, 1991). Thus, identification of threshold size is challenging in this species. In summary, although molecular disruptions that cause precocious metamorphosis or supernumerary molts have been identified, none of these answer the question of how size is assessed so that metamorphosis occurs at the correct speciesspecific body size.

In this study, we sought to examine how M. sexta larvae assess their body size to initiate metamorphosis. Our approach was to utilize two distinct methods to generate a wide range of body sizes at the end of the fourth instar (penultimate instar under laboratory conditions): nutrient-deprivation and hypoxia. As mentioned above, nutrient-deprivation is the standard way by which threshold size has been determined in $M$. sexta. Aside from nutrient deprivation, previous studies have shown that hypoxia can also stunt growth in most insect species, including M. sexta (Callier and Nijhout, 2011; Frazier et al., 2001; Greenberg and Ar, 1996). We therefore exposed larvae at different instars to hypoxic conditions to generate a range of fourth instar larval sizes. We then sought to identify traits that correlate with the attainment of the threshold size under both nutrient-deprivation and hypoxia conditions to narrow down the potential molecular regulators involved in threshold size determination.

We found that nutrient deprivation at different times of larval development leads to distinct threshold sizes and found that the relative muscle/integument mass is correlated with the attainment of the threshold size. We also show that myo is expressed differentially in muscles/integuments of pre-threshold size larvae relative to that of post-threshold size larvae. Because knockdown of gene expression in vivo is not possible in $M$. sexta, we explored the function of myo in another holometabolous insect, the flour beetle T. castaneum, where RNAi is possible (Tomoyasu and Denell, 2004). We demonstrate that myo dsRNA-injected larvae continue to molt into supernumerary larval instars and never initiate metamorphosis even after surpassing the threshold size, indicating that myo is the signal by which body size is sensed and that triggers the transition between growth and metamorphosis.

\section{RESULTS}

\section{Effect of nutrient deprivation on the threshold size}

Under standard laboratory rearing conditions, $M$. sexta larvae invariably undergo five larval instars. This precludes us from determining the threshold size. Thus, the standard way to 
169

170

171

172

173

174

175

176

177

178

179

180

181

182

183

184

185

186

187

188

189

190

191

192

193

194

195

196

197

198

199

determine the threshold size is to temporarily starve larvae or to feed larvae a diet containing a reduced amount of protein to generate large variability in size (Grunert et al., 2015; Nijhout, 1975). Here, we used both methods to determine the threshold size. First, larvae were fed an experimental diet with reduced levels of protein ( $40 \%$ diet) starting the second instar. These larvae had a threshold size of approximately $0.85 \mathrm{~g}$ (Fig. 1A, 1C). In contrast, shifting the timing of nutrient deprivation altered the threshold size. When larvae were starved during the third instar after one day of feeding, or when third instar larvae were placed on $40 \%$ diet temporarily and returned to the normal diet at the end of the third instar, larvae had a reduced threshold size: Larvae on the $40 \%$ diet only in the third instar and larvae starved in the third instar had a threshold size of approximately $0.65 \mathrm{~g}$ and $0.75 \mathrm{~g}$, respectively (Fig. 1A, C). Thus, the timing of nutrient deprivation appears to affect the threshold size.

\section{Hypoxia generates supernumerary larvae}

Hypoxia has also been demonstrated to slow down the growth rate (Callier et al., 2013; Harrison et al., 2015). Therefore, we exposed third instar larvae to hypoxia to see if we could generate smaller larvae. After exposure to hypoxia, fourth instar larvae were returned to normoxic conditions and weighed daily until the beginning of the wandering stage, which indicates the onset of metamorphosis (Fig. S1A). These larvae grew to a smaller size at the end of the fourth instar and had two distinct fates: at the end of the fifth instar, $40 \%$ of the larvae $(n=61)$ underwent a supernumerary larval molt, and the remaining larvae entered the wandering stage $(n=90)$ (Fig. S1B). Larvae exposed to hypoxic conditions during the third instar that wandered at the end of the fifth instar had a fourth instar feeding period of 3.0 days, similar to the average feeding time of normoxic control larvae, which took 2.9 days (Fig. S2). In contrast, larvae that underwent a supernumerary molt at the end of the fifth instar had a significantly reduced fourth instar feeding period of 2.0 days (Fig. S2; One-way ANOVA: F(2,159)=125.556, $\mathrm{p}<0.0001)$. Thus, the feeding duration of the fourth instar is a significant predictor of the nature of the molt that occurs at the end of the fourth instar.

The weights at the end of the fourth instar are highly predictive of the fate of the molt, indicating that the threshold size checkpoint occurs at the end of the fourth instar, similar to the nutrient-deprivation conditions (Fig. S3A, B). The mass at the end of the third instar is not predictive of the mass at the end of the fourth instar (Fig. S3C). In the hypoxia-treated larvae, the 
threshold size is approximately $0.65 \mathrm{~g}$ (Fig. 2, S3B, C), similar to that of larvae reared under normoxia/low-nutrient conditions only during the third instar. on the timing of nutrient deprivation, we reared fourth instar larvae in hypoxic conditions instead of third instar larvae and observed whether the threshold size changed. Under these conditions, the threshold size was also approximately $0.65 \mathrm{~g}$, similar to the threshold size of larvae exposed to hypoxia during the third instar (Fig. 2A, C). Thus, it appears that the timing of hypoxia treatment does not alter the threshold size.

\section{Muscle mass is correlated with threshold size attainment}

Because we determined that distinct nutritional conditions can affect the threshold size, we hypothesized that the size of a growing body part might contribute to threshold size determination. Two tissues that grow extensively throughout an instar are the fat body and the muscles. We therefore determined the muscle/integument mass and fat body mass for the third instar hypoxia-treated larvae and larvae fed a $40 \%$ diet throughout much of the early larval instars as these two treatments reliably generate fourth instar larvae with masses close to the threshold size and lead to distinct threshold sizes. We also determined the muscle/integument mass and fat body mass at the end of the third and fourth instar larvae reared under normoxia/normal diet conditions.

Although the third and fourth instar normoxia/normal diet-fed larvae are at the extreme ends of the size range, the hypoxia-treated and normoxia/normal diet-fed larvae appear to have

222 similarly in hypoxia-treated and normoxia/normal diet-fed larvae (Fig. 3A). In contrast, the

223 larvae fed a 40\% diet had smaller relative mass of the muscles/integuments than hypoxia-treated

224 and normoxia/normal diet-fed larvae (Fig. 3A). We found that the relative mass of the

225 muscles/integuments was larger for hypoxia-treated larvae than larvae fed a 40\% diet, and a

226 significant wet mass $X$ treatment interaction was observed (ANCOVA: $F(1,39)=13.016$, $227 \mathrm{p}<0.001$; Fig. 3A). Intriguingly, the mass of the muscles/integuments at the threshold size for 228 both the hypoxia-treated larvae and the $40 \%$ diet-fed larvae were similar. In contrast, fat body mass scaled with whole body mass similarly in both environmental conditions, and there was no significant wet mass $X$ treatment interaction (ANCOVA: $F(1,39)=0.0142, p=0.906$; Fig. 3B). 
231 Together, these results suggest that muscle/integument mass is correlated with attainment of

232 threshold size.

myo expression in muscles is correlated with the attainment of threshold size

Given that the integument/muscle mass was correlated with the attainment of threshold size, we hypothesized that the growing muscles might produce a factor that signals the swtich from the growth phase to the metamorphic onset. We reasoned that this signal would have to be expressed differentially between pre-threshold size and post-threshold size larvae reared under normoxia/normal diet, hypoxia and nutrient-deprivation conditions. We also suspected that the signal might be secreted in order to be able to communicate with the rest of the body. Finally, we reasoned that this factor would need to be expressed in the muscle. Given that Myo is expressed in the muscles (Augustin et al., 2017; Lo and Frasch, 1999) and its removal leads to supernumerary molts in hemimetabolous insects (Kamsoi and Belles, 2019), we suspected that Myo might be a potential candidate factor. We identified three Activin ligand genes in the $M$. sexta genome. The predicted amino acid sequences were used to verify the identity of the Myocoding gene (Fig. S4). The alignment of M. sexta Myo is shown in Fig. S5.

We first examined the myo expression in the anterior portion (containing the brain and thoracic structures) of second, third and fourth instar larvae undergoing a molt using quantitative RT-PCR (qPCR). We found that the expression of myo increased with each molt (Fig. 4A). To examine how the tissue-specific expression of myo changes between larvae at the end of the third instar (under the threshold size) and those at the end of the fourth instar (above the threshold size), the muscles, fat body and CNS were dissected from larvae reared under standard rearing conditions. We observed a significant increase in the expression of myo in the muscles (Student's t-test: $\mathrm{t}(6)=2.82, \mathrm{p}<0.05)$, but no significant increase was detected in the fat body (Student's ttest: $\mathrm{t}(6)=0.637, \mathrm{p}=0.55$ ) and the CNS (Student's t-test: $\mathrm{t}(5)=0.417, \mathrm{p}=0.69)$ (Fig. 4B-D). Thus,

256 an increase in myo expression in the muscle was correlated with the attainment of threshold size 257 in normoxia/normal diet-fed larvae.

To further explore this correlation, we examined the tissue-specific expression of myo in

259 muscles, fat body and CNS of the pre- and post-threshold size larvae in both hypoxia-treated and $40 \%$ diet-fed fourth instar larvae. We found significantly higher expression of myo in muscles of 
262 fed larvae (Fig. 5; Student's t-test: $\mathrm{t}(8)=3.65, \mathrm{p}<0.01$ for hypoxia; $\mathrm{t}(8)=3.98, \mathrm{p}<0.005$ for $40 \%$

263 diet). In the fat body, significantly higher expression of myo was observed in the post-threshold

264 size fat body of $40 \%$ diet fed larvae relative to pre-threshold size larvae (Student's t-test:

$265 \mathrm{t}(7)=3.41, \mathrm{p}<0.05)$. However, in hypoxia-treated larvae, no statistically significant differences

266 were observed although post-threshold size larvae tended to have higher myo expression

267 (Student's t-test: $\mathrm{t}(8)=1.43, \mathrm{p}=0.19)$. In contrast, no difference in myo expression was observed

268 in the CNS of pre- or post-threshold size larvae under both hypoxia and $40 \%$ diet conditions

269 (Fig. 5; Student's t-test: $\mathrm{t}(8)=1.01, \mathrm{p}=0.34$ for hypoxia; $\mathrm{t}(8)=1.09, \mathrm{p}=0.31$ for $40 \%$ diet).

270 To confirm the correlation between myo in the muscles and the atainment of threshold

271 size, we isolated muscles and fat body from hypoxia-treated and 40\% diet-fed larvae weighing

272 between $0.7 \mathrm{~g}$ and $0.8 \mathrm{~g}$. In this weight range, hypoxia-treated larvae are above the threshold size

273 whereas $40 \%$ diet-fed larvae are below the threshold size. We found that myo expression was

274 significantly higher in the muscles of hypoxia-treated larvae than that of $40 \%$ diet-fed larvae

275 (Fig. 5C; Student's t-test: $\mathrm{t}(6)=4.36, \mathrm{p}<0.005)$. In contrast, myo expression in the fat body did not

276 differ significantly (Fig. 5C; $\mathrm{t}(6)=1.05, \mathrm{p}=0.34$ ). Taken together, the attainment of threshold size

277 is consistently correlated with elevated myo expression in the muscles/integuments under all

278 experimental conditions.

\section{JH does not shift the threshold size}

Previous studies have suggested that a decline in JH may underlie threshold size attainment in other insects (Chafino et al., 2019). To determine whether JH affects threshold size attainment in M. sexta, larvae were placed in hypoxic conditions during the third instar until

$284 \mathrm{HCS}$ and then treated with $10 \mu \mathrm{g}$ methoprene, a JH analog, two days later. These larvae typically underwent a molt approximately 1-3 days after treatment. The fates of the larvae were tracked to see if they underwent an extra molt or initiated wandering. We found that the threshold size was around $0.67 \mathrm{~g}$ (Fig. S6A). This is similar to that of the hypoxia-treated larvae without methoprene treatment, indicating that methoprene treatment does not shift the threshold size. All methoprene-treated larvae, including those that were destined to undergo metamorphosis at the end of the fifth instar, showed complete loss of melanic markings that are normally visible in the

291 fifth instar (Fig. S6B, C), suggesting that JH signaling was active. Thus, while methoprene

292 clearly had the expected effect on the body coloration, it did not alter the threshold size. 
293

294

295

296

297

298

299

300

301

302

303

304

305

306

307

308

309

310

311

312

313

Together, these data indicate that myo expression is correlated with the attainment of threshold size, and that methoprene treatment before threshold size attainment is insufficient to shift the threshold size.

\section{myo knockdown in Tribolium leads to indefinite molts}

Because gene manipulation is not possible in M. sexta, we explored the role of myo in $T$. castaneum, a species where RNAi is possible. In Tribolium, even under normal rearing conditions, the total number of instars can vary, and like in M. sexta, the timing of metamorphosis is determined by a drop in $\mathrm{JH}$. In our laboratory, the GA-1 strain typically undergoes 7 or 8 instars. A recent study has shown that by day 1 of the final instar, the decision to pupate and hence the threshold size has already been reached (14). We weighed larvae 1 day after a molt and determined their fates. When larvae weighed above $2.1 \mathrm{~g}, 50 \%$ of the larvae initiated metamorphosis (Fig. 6A), indicating that if larvae are above $2.1 \mathrm{~g}$, they have already reached the threshold size.

The myo homolog was identified in the Tribolium genome and clusters with other Myo homologs in other insects (Fig. S4, S5). We examined the expression of myo in freshly molted sixth instar (below the threshold size) or freshly molted final instar larvae weighing at least 2.2 mg (above the threshold size). To do this, we removed the gut and the fat body and examined the expression in the rest of the body (containing the CNS, muscles and integuments). We found a small but significant increase in myo expression in the seventh instar larvae (Fig. 6B; Student's ttest: $\mathrm{t}(6)=3.02, \mathrm{p}<0.05)$.

To functionally characterize the role of myo, we injected $a m p^{r}$ and myo dsRNA into larvae. Knockdown verification experiments demonstrated that myo dsRNA successfully knocked down the expression of myo (Fig. 7A). Eleven out of $18 a m p^{r}$ RNAi larvae injected in the sixth instar pupated after the seventh instar while four larvae pupated after the eighth instar (Table 1). $a m p^{r}$ dsRNA-injected seventh instar larvae all underwent pupation without a larvallarval molt ( $\mathrm{n}=7$; Table 1). Knockdown of myo did not induce visible morphological changes. However, larvae injected with myo dsRNA as sixth instars continued to molt indefinitely, and those injected as seventh instars never molted; none of these larvae ever entered the prepupal stage ( $\mathrm{n}=18$; Table 1). In addition, the intermolt period was significantly increased (Fig. 7B). Overall, the larval duration of $a m p^{r}$ dsRNA-injected larvae was 12 days whereas myo dsRNA- 
324 injected larvae stayed at the larval stage for up to 7 months before dying (Fig. 7C). When a

325 subset of the larvae was weighed and their fates were assessed, we found that myo dsRNA-

326 injected larvae grew at a slower rate than the $a m p^{r}$ dsRNA-injected larvae (Fig. 7D). However,

327 the myo dsRNA-injected larvae continued to molt as supernumerary larvae even when they

328 weighed more than the mass of irreversible pupal commitment (i.e. $2.1 \mathrm{mg}$ ) one day after the

329 molt and should have pupated (Fig. 7E). In contrast, $a m p^{r}$ dsRNA-injected larvae weighing more

330 than $2.1 \mathrm{mg}$ one day after the molt pupated at the end of the instar (Fig. 7E). These observations

331 indicate that myo dsRNA-injected larvae continued to undergo supernumerary molts even after

332 reaching the size when larvae are normally pupally committed, thus supporting the idea that Myo

333 is the signal that mediates the switch between growth and metamorphosis.

\section{DISCUSSION}

In this study, we investigated the mechanism by which insects sense their body size and initiate the switch from larval growth to metamorphosis. We found that hypoxia-treatment and a nutrient-deficient diet can both alter threshold sizes in $M$. sexta. The muscle/integument mass was found to be correlated with threshold size: although the relative size of muscles/integuments was greater in hypoxia-treated larvae than that in nutrient-deprived larvae, the same absolute muscle/integument mass was observed at their respective threshold sizes. We found that myo expression increases significantly in the muscles during development. Moreover, in the muscles,

342 the expression of myo was significantly higher in post-threshold size larvae than in pre-threshold 343 size larvae under both hypoxia and $40 \%$ nutrient treatments. myo RNAi knockdown in $T$.

344 castaneum, led to permanent indefinite supernumerary larval-larval molts even in larvae that 345 were larger than the threshold size. Based on these findings, we propose that Myo is the signal 346 by which larvae can assess their body size.

\section{A nutrient-dependent pathway regulates the threshold size}

Because the threshold size sets the number of larval instars, the size at metamorphosis

350 and the size of the adult, the threshold size is arguably the most important determinant of final

351 body size. In this study, we found that prolonged exposure to reduced nutrient-diet increases the

352 threshold size (Fig. 1). When larvae were nutritionally deprived during the third instar, the 353 threshold size ranged between $0.65 \mathrm{~g}$ to $0.75 \mathrm{~g}$. However, when larvae were fed a $40 \%$ diet 
throughout much of the larval instar, the threshold size increased to $0.85 \mathrm{~g}$. Poor nutrient

355 conditions throughout much of the growth period, or recovery from starvation during the third

356 instar, therefore increase the threshold size, suggesting that a nutrient-dependent process likely

357 contributes to the determination of threshold size. In contrast, hypoxia treatment does not reduce

358 the threshold size as larvae reared in hypoxic conditions in either the third or the fourth instar

359 had a threshold size of approximately $0.65 \mathrm{~g}$, similar to larvae that had been fed a $40 \%$ diet

360 during the third instar. These findings indicate that $0.65 \mathrm{~g}$ may be the "actual" threshold size

361 under normal nutrient conditions.

362 We discovered that the mass of the muscles/integuments is correlated with threshold size

363 in both hypoxia-treated larvae and nutrition-deprived larvae. The relative muscle/integument

364 mass at threshold size of hypoxia-treated larvae is greater than the relative muscle/integument

365 mass in nutrition-deprived larvae, which have a larger threshold size. Furthermore, the

366 muscle/integument masses at the threshold size of hypoxia-treated and nutrient-deprived larvae

367 are similar. Such correlations are not observed in the fat body mass. These observations indicate

368 that muscle/integument mass serves as a good proxy for body size and the threshold size. Since

369 only the muscle showed consistent increase of myo expression in post-threshold size larvae, we

370 think that the expression of myo in the muscle is the key signal by which insects assess their

371 body size.

Myo mediates the transition between growth and metamorphosis

Using T. castaneum, we found that myo RNAi leads to indefinite larval-larval molts. The

376 However, these larvae continued to molt as larvae even when they had reached a mass that

377 normally would have initiated prepupal development. Thus, these larvae clearly had reached the

378 threshold size but were unable to molt into the final instar.

379 Based on our findings, we propose that Myo couples the attainment of threshold size to

380 the initiation of metamorphosis (Fig. 8). We found that myo expression in the muscles increases

381 during the growth phase and is consistently expressed at significantly higher levels in muscles of

382 post-threshold size larvae. The muscle size is a reliable proxy for body size, and the correlated

383 increase in myo serves as a signal of body size. Thus, we propose that Myo levels in muscles

384 provide a molecular readout of body size. Upon the attainment of a threshold size, the muscles 
385

386

387

388

389

390

391

392

393

394

395

396

397

398

399

400

401

402

403

404

405

406

407

408

409

410

411

412

413

414

415

produce enough Myo to signal to the neuroendocrine center to switch from the growth phase to initiate the physiological processes of metamorphosis. At this point, we do not yet know if Myo levels in the hemolymph stimulate the neuroendocrine glands directly or via another relay system like the nervous system. For example, Myo has been shown to inhibit neuromuscular junction development and synaptic transmission in D. melanogaster (Augustin et al., 2017). Thus, it is possible that the signals are transmitted neuronally to the brain or to the corpora cardiaca/corpora allata.

Taken together, our study demonstrates that one single signaling molecule couples growth to the initiation of metamorphosis and ensures that metamorphosis is triggered when a larva passes the threshold size. Such a model would explain the sharp and precise transition in fates of larvae at the threshold size.

myo RNAi has also been conducted in hemimetabolous insects: In both the cricket, $G$. bimaculaus, and the cockroach, $B$. germanica, silencing myo leads to multiple supernumerary nymphal molts, often leading to larger body masses (Ishimaru et al., 2016; Kamsoi and Belles, 2019). Thus, in both hemimetabolous and holometabolous insects, myo may act as the switch that mediates allows juveniles to shift from the growth phase to the reproductive phase once the animal has reached a specific size threshold. It will therefore be of interest to determine whether a threshold size can be identified in hemimetabolous insects.

Finally, JH-deficient larvae of T. castaneum and B. mori must undergo at least three larval molts to produce an unknown "competence factor" that allows them to initiate metamorphosis (Daimon et al., 2015; Smykal et al., 2014). Whether or not Myo is related to the "competence factor" is unclear at this point.

\section{JH does not affect threshold size of $\mathrm{M}$. sexta}

When we applied JH during the fourth instar of $M$. sexta, we observed that the threshold size could not be shifted (Fig. S6). Thus, we believe that JH is not the primary regulator of threshold size in $M$. sexta. Instead, JH is a downstream effector that mediates the decision to metamorphose post-threshold size attainment.

A previous study has suggested that threshold size in T. castaneum might be regulated by JH (Chafino et al., 2019). However, in that study, the threshold size was identified by starving larvae at various sizes during the final instar and identifying the size above which the larva 
initiates metamorphosis. Whether this size checkpoint is equivalent to the threshold size checkpoint remains unclear. While the clearance of $\mathrm{JH}$ is necessary to initiate metamorphosis, the threshold size assessment must occur earlier. Myo has been shown to act upstream of JH signaling in hemimetabolous insects: myo knockdown leads to an increase in jhamt expression in the corpora cardiaca/corpora allata (Kamsoi and Belles, 2019). Thus, Myo is likely the threshold size determinant that ultimately causes a drop in JH titer. larvae. In T. castaneum, feeding larvae on flour that has been diluted to $20 \%$ during the fifth instar leads to a lack of molt and eventual metamorphosis, similar to a bail-out response seen in other beetles (Nagamine et al., 2016; Shafiei et al., 2001; Terao et al., 2015). M. sexta does not appear to exhibit this type of bail-out response as they either molt or die when nutrients are removed. The bail-out response seen in T. castaneum likely represents an adaptive response that is absent in M. sexta.

Conserved functions of Myo in T. castaneum and other insects

In addition to the role of Myo during the threshold size checkpoint, myo knockdown in $T$. castaneum revealed additional functions of Myo that are conserved across insects. When myo was knocked down in $T$. castaneum, the intermolt period was dramatically increased, with some larvae taking a month to molt. Normally, the intermolt period is around five days. Thus, myo knockdown delays the onset of a molt, presumably through a decrease in ecdysteroidogenesis. Activin signaling has previously been shown to affect ecdysteroidogenesis in both B. germanica and D. melanogaster (Gibbens et al., 2011; Santos et al., 2016). Thus, the role of Activin signaling on ecdysteroidogenesis appears to be conserved across various insect species. that it promotes growth. This growth promoting function of Myo appears to be conserved in most insects (Ishimaru et al., 2016; Kamsoi and Belles, 2019). The only exception is found in $D$. melanogaster, where an opposite effect is observed: In D. melanogaster, knockdown of myo in the muscles leads to a larger larval size whereas overexpression of myo in the muscles leads to smaller larval body size without affecting the developmental time (Augustin et al., 2017). In this species, the functions of TGF-beta ligands appear to be switched with Activin-beta playing a growth promoting role, similar to the growth-promoting role of myo in other insect species: the 
447 loss-of-function mutation in the Activin-beta gene leads to smaller muscle and adult body size in

448 Drosophila without affecting the timing of metamorphosis (Moss-Taylor et al., 2019). Thus, the

449 growth promoting roles of TGF-beta ligands may have been switched in the lineage leading to $D$.

450 melanogaster. Such switches in function have also been reported for other TGF-beta ligands

451 (Namigai and Suzuki, 2012).

Implications for mammalian growth

454 Body size regulation in insects also has many parallels to size regulation in mammals.

455 Puberty in mammals, like metamorphosis in insects, is initiated upon reaching a specific

456 threshold size, and its onset is affected by body size and nutritional status (Hirsch and Batchelor,

457 1976). Obesity in children leads to precocious pubertal onset, a public health issue that is

458 affecting US youths (Burt Solorzano and McCartney, 2010; Euling et al., 2008). While being

459 overweight can lead to precocious puberty, we do not know if the threshold size itself is shifted

460 under altered nutritional regimes. Based on our study, we hypothesize that the precocious

461 pubertal onset may be a product not only of precocious attainment of body size but potentially

462 also of an adjustment of the threshold size itself. Whether the vertebrate homologs of Myo,

463 BMP-11/GDF-8, mediate the transition between pre-pubertal growth and puberty has not been

464 clearly demonstrated although a polymorphism in GDF-8 has recently been shown to delay

465 puberty in cows (Cushman et al., 2015). Additional studies are necessary to demonstrate whether

466 an evolutionarily conserved mechanism is involved in the regulation of determinate growth.

468 CONCLUSION

469 In this study, we sought to identify the mechanism by which larvae assess their body size.

470 Specifically, we investigated mechanism by which larvae sense the threshold size, the first size

471 check point that determines the timing of metamorphosis. Muscle growth and the expression of

472 the myo in the muscles are linked to the attainment of the threshold size, the earliest body size

473 checkpoint for metamorphosis. 


\section{METHODS}

\section{Animal rearing}

478 Wildtype M. sexta were obtained from Carolina Biological Supply Company. Larvae were fed a 479 standard artificial diet as described previously (Kemirembe et al., 2012) unless otherwise noted. 480 Larvae were raised in individual plastic cups at $26.5^{\circ} \mathrm{C}$ and a $16: 8 \mathrm{hr}$ light:dark cycle. Larvae 481 were kept in $1 \mathrm{oz}$ soufflé cups until the end of the fourth instar, when they were moved to a $5 \mathrm{oz}$ 482 soufflé cup. The end of an instar can be clearly identified in this species when the head capsule 483 begins to slip (denoted "HCS" for head capsule slippage) as the larva initiates a molt. Wildtype 484 GA-1 strain Tribolium castaneum beetles were raised on organic whole wheat flour supplemented with $5 \%$ nutritional yeast and $0.5 \%$ fumagilin at $29.5^{\circ} \mathrm{C}$ and $\sim 55 \%$ humidity.

Hypoxia treatment

488 M. sexta larvae subjected to hypoxia treatments were moved to an airtight cell culture chamber immediately after molting into either the third or the fourth instar and kept in cups with multiple holes in the lid. A 5\% oxygen/carbon dioxide mixture was sent into the chamber and oxygen

491 levels were kept at approximately $4 \pm 1 \%$ throughout the experiment. Larvae were removed from 492 the chamber at the end of the instar, after which the artificial diet was replaced with fresh diet, 493 and multi-holed lids were exchanged for single-holed lids.

Effects of nutrients on threshold size

496 To assess the effects of nutrition on growth and threshold size, larvae were removed from the 497 standard artificial diet and placed onto experimental diets or subjected to starvation conditions at 498 the onset of the third or fourth instar. The experimental diet contained $40 \%$ the amount of dietary 499 protein compared to the standard diet by having reduced levels of wheat germ and casein (Table 500 S1). Larvae subjected to starvation conditions were removed from the standard diet 24 hrs after 501 molting into the third instar and placed onto a moistened Kimwipe, which served as a water 502 source. All larvae were returned to the standard artificial diet when they initiated HCS. 
505 To test the effect of $\mathrm{JH}$ on threshold size, third instar larvae were hypoxia-treated at the onset of

506 the third instar as described above. Two days after the onset of third instar HCS, $1 \mu 1$ of

507 methoprene (Sigma) dissolved in acetone $(10 \mu \mathrm{g} / \mu \mathrm{l})$ was applied topically on the dorsal side of

508 the fourth instar. These larvae were weighed at fourth instar HCS and subsequently tracked to

509 see if they entered the wandering stage - an early indication of the onset of metamorphosis - or

510 initiated another molt to determine the threshold size.

512 Threshold size determination

513 To determine how varying growth conditions altered the threshold size, $M$. sexta larvae were

514 subjected to varying nutritional or hypoxic conditions, and their growth was followed. Subjecting

515 larvae to these sub-optimal growth conditions generates larvae above and below threshold size at

516 the end of the fourth instar, and threshold size can be determined by plotting their developmental

517 fate against their mass at that time. Larvae were moved onto experimental diets at the onset of

518 the third or fourth instar or subjected to starvation or hypoxic conditions as described above.

519 Larvae were checked daily, starting on the day of HCS into the fourth or fifth instar, for third or

520 fourth instar hypoxia-treated larvae, respectively. Observations continued until larvae initiated

521 HCS into a supernumerary instar or exhibited signs of metamorphosis, as indicated by a purging

522 of the gut contents and development of a darkened dorsal vessel. The percentage of larvae that

523 entered final larval instars was plotted against mass at the time of the fourth HCS and fitted to a

524 sigmoidal growth curve. For each treatment, the mass at which $50 \%$ of the larvae entered the

525 final instar was determined to be the threshold size.

526

527 Muscle and fat body mass

528 After the larvae were weighed, they were dissected in $1 \mathrm{X}$ phosphate-buffered saline (PBS; 0.15

$529 \mathrm{M} \mathrm{NaCl}, 0.0038 \mathrm{M} \mathrm{NaH}_{2} \mathrm{PO}_{4}, 0.0162 \mathrm{M} \mathrm{Na}_{2} \mathrm{HPO}_{4} ; \mathrm{pH}$ 7.4). The gut, central nervous system

530 (CNS) and other tubular structures were removed. The fat body was then carefully removed and

531 placed on an aluminum foil, dried at $60^{\circ} \mathrm{C}$ for at least $48 \mathrm{hrs}$, and then weighed. The rest of the

532 larval body, representing the combination of muscles and integuments, was also dried and

533 weighed. 
536 To determine the expression of myo at the end of various instars, RNA was isolated from the

537 anterior half of the larva (including the head and the thorax) at the onset of HCS when the head

538 capsule was still fluid filled and mandibles were white; three biological replicates were created

539 for each time point. To determine the expression of myo in pre-threshold size and post-threshold

540 size larvae, larvae were either placed on $40 \%$ diet once they molted into the second instar or

541 placed in hypoxia conditions for the duration of the third instar. Muscles, fat body and CNS were

542 dissected from Manduca at the onset of HCS in the fourth instar in 1X PBS. To isolate RNA,

543 tissues were homogenized in $500 \mu \mathrm{L}$ of TRIzol reagent (Thermo Fisher). After extracting RNA

544 using chloroform, the RNA was treated with DNAse (RQ1 RNase-Free DNase, Promega) to

545 remove remaining traces of genomic DNA. The First Strand cDNA Synthesis Kit (Thermo

546 Fisher) was used to convert $1 \mu \mathrm{g}$ of RNA to cDNA via reverse transcription.

Quantitative polymerase chain reaction ( $q P C R)$

549 SYBR Green Supermix (Bio-Rad) and qPCR primers (Table 2) were used for qPCR. To measure myo expression, primers targeting the Manduca homolog of the Drosophila myo gene (Genbank accession no.XM_030169402.1), were designed. Each replicate was assayed in triplicate with no-template controls. $R p L 17 A$ was used as an internal control gene, and a standard curve method

553 was used to analyze the data. JMP (SAS Institute, Cary, NC) was used for statistical analyses.

Double-stranded RNA (dsRNA) synthesis

556 T. castaneum myo gene (Genbank accession nr: XM_961726.3) was amplified using the primer

557 set listed in Supplemental Table 1. The PCR product was inserted into a pCR4 TOPO vector

558 (Thermo Fisher Scientific) following the manufacturer's instructions. Plasmid DNA from

559 transformed E. coli cells were isolated according to the QIAprep Spin Miniprep Kit protocol

560 (Qiagen). A restriction reaction was then set up to linearize the plasmid DNA. Using $1 \mu \mathrm{g}$ of

561 linearized plasmid as a template, ssRNA was then synthesized using the MEGAscript T3 and T7

562 kits. The synthesized ssRNA was cleaned using phenol/chloroform extraction and annealed to 563 make a $2 \mu \mathrm{g} / \mu 1$ solution as described previously (Hughes and Kaufman, 2000). Proper annealing 564 was checked using gel electrophoresis. 
566 Microinjection of T. castaneum larvae

567 Before injection with dsRNA, sixth and seventh instar T. castaneum larvae were first

568 anesthetized on ice. A $10 \mu \mathrm{L}$ glass capillary needle was used to inject $0.5 \mu \mathrm{L}$ of dsRNA into

569 seventh instar larvae and $0.25 \mu \mathrm{L}$ into sixth instar larvae. Control animals were also injected with

570 the same volume of bacterial ampicillin-resistance $\left(a m p^{r}\right)$ dsRNA.

571

572 Knockdown verification

573 In order to verify proper knockdown of myo, day 0 sixth instar $T$. castaneum larvae were injected

574 with either myo or $a m p^{r}$ dsRNA. RNA from three day 4 sixth instar larvae were collected and

575 processed as outlined above. After converting $1 \mu \mathrm{g}$ of RNA into cDNA, PCR was run using myo

576 and rp49 primers listed in Table 2. For $r p 49,20,25$ and 30 cycles were run; for myo, 30, 35 and

57740 cycles were run.

578

579 Competing interests

580 The authors declare that they have no competing interests

581

$582 \quad$ Funding

583 This work was funded by grants from Wellesley College and the National Science Foundation

584 IOS-1354608 to Y.S.

585

586

\section{Acknowledgements}

587 We thank Drs. Kimberly O’Donnell, Louise Darling, Melissa Beers and Julie Roden for their

588 helpful advice and discussions. We also thank the members of the Suzuki lab and Heidi Park for

589 their support and comments on the manuscript. 


\section{REFERENCES}

Augustin, H., McGourty, K., Steinert, J.R., Cocheme, H.M., Adcott, J., Cabecinha, M., Vincent, A., Halff, E.F., Kittler, J.T., Boucrot, E. and Partridge, L. 2017. Myostatin-like proteins regulate synaptic function and neuronal morphology. Development 144, 2445-2455.

Burt Solorzano, C.M. and McCartney, C.R. 2010. Obesity and the pubertal transition in girls and boys. Reproduction 140, 399-410.

Callier, V. and Nijhout, H.F. 2011. Control of body size by oxygen supply reveals sizedependent and size-independent mechanisms of molting and metamorphosis. Proc. Natl. Acad. Sci. USA 108, 14664-14669.

Callier, V., Shingleton, A.W., Brent, C.S., Ghosh, S.M., Kim, J. and Harrison, J.F. 2013. The role of reduced oxygen in the developmental physiology of growth and metamorphosis initiation in Drosophila melanogaster. J. Exp. Biol. 216, 4334-4340.

Chafino, S., Urena, E., Casanova, J., Casacuberta, E., Franch-Marro, X. and Martin, D. 2019. Upregulation of E93 gene expression acts as the trigger for metamorphosis independently of the threshold size in the beetle Tribolium castaneum. Cell Rep. 27, 1039-1049 e1032.

Cheng, C., Ko, A., Chaieb, L., Koyama, T., Sarwar, P., Mirth, C.K., Smith, W.A. and Suzuki, Y. 2014. The POU factor ventral veins lacking/Drifter directs the timing of metamorphosis through ecdysteroid and juvenile hormone signaling. PLoS Genet. 10, e1004425.

Cushman, R.A., Tait, R.G., Jr., McNeel, A.K., Forbes, E.D., Amundson, O.L., Lents, C.A., Lindholm-Perry, A.K., Perry, G.A., Wood, J.R., Cupp, A.S., Smith, T.P., Freetly, H.C. and Bennett, G.L. 2015. A polymorphism in myostatin influences puberty but not fertility in beef heifers, whereas micro-calpain affects first calf birth weight. J. Anim. Sci.93, 117-126.

Daimon, T., Kozaki, T., Niwa, R., Kobayashi, I., Furuta, K., Namiki, T., Uchino, K., Banno, Y., Katsuma, S., Tamura, T., Mita, K., Sezutsu, H., Nakayama, M., Itoyama, K., Shimada, T. and Shinoda, T. 2012. Precocious metamorphosis in the juvenile hormone-deficient mutant of the silkworm, Bombyx mori. PLoS Genet. 8, e1002486

Daimon, T., Uchibori, M., Nakao, H., Sezutsu, H. and Shinoda, T. 2015. Knockout silkworms reveal a dispensable role for juvenile hormones in holometabolous life cycle. Proc. Natl. Acad. Sci. USA 112, E4226-4235. 
Euling, S.Y., Herman-Giddens, M.E., Lee, P.A., Selevan, S.G., Juul, A., Sorensen, T.I., Dunkel, L., Himes, J.H., Teilmann, G. and Swan, S.H. 2008. Examination of US puberty-timing data from 1940 to 1994 for secular trends: panel findings. Pediatrics 121, S172-191.

Frazier, M.R., Woods, H.A. and Harrison, J.F. 2001. Interactive effects of rearing temperature and oxygen on the development of Drosophila melanogaster. Physiol. Biochem. Zool. 74, 641-650.

Gibbens, Y.Y., Warren, J.T., Gilbert, L.I. and O'Connor, M.B. 2011. Neuroendocrine regulation of Drosophila metamorphosis requires TGFbeta/Activin signaling. Development 138, 2693-2703.

Greenberg, S., Ar, A., 1996. Effects of chronic hypoxia, normoxia and hyperoxia on larval development in the beetle Tenebrio molitor. J. Insect Physiol. 42, 991-996.

Grunert, L.W., Clarke, J.W., Ahuja, C., Eswaran, H. and Nijhout, H.F. 2015. A quantitative analysis of growth and size regulation in Manduca sexta: the physiological basis of variation in size and age at metamorphosis. PLoS One 10, e0127988.

Harrison, J.F., Shingleton, A.W . and Callier, V. 2015. Stunted by Developing in Hypoxia: Linking Comparative and Model Organism Studies. Physiol Biochem Zool 88, 455-470.

Hirsch, J. and Batchelor, B., 1976. Adipose tissue cellularity in human obesity. Best Pract. Res. Clin. Endocrinol. Metab. 5, 299-311.

Hughes, C.L. and Kaufman, T.C. 2000. RNAi analysis of Deformed, proboscipedia and Sex combs reduced in the milkweed bug Oncopeltus fasciatus: novel roles for Hox genes in the Hemipteran head. Development 127, 3683-3694.

Ishimaru, Y., Tomonari, S., Matsuoka, Y., Watanabe, T., Miyawaki, K., Bando, T., Tomioka, K., Ohuchi, H., Noji, S. and Mito, T. 2016. TGF-beta signaling in insects regulates metamorphosis via juvenile hormone biosynthesis. Proc. Natl. Acad. Sci. USA 113, 56345639.

Kamsoi, O. and Belles, X. 2019. Myoglianin triggers the premetamorphosis stage in hemimetabolan insects. FASEB J. 33, 3659-3669.

Kemirembe, K., Liebmann, K., Bootes, A., Smith, W.A. and Suzuki, Y. 2012. Amino acids and TOR signaling promote prothoracic gland growth and the initiation of larval molts in the tobacco hornworm Manduca sexta. PLoS One 7, e44429 
Kingsolver, J.G. 2007. Variation in growth and instar number in field and laboratory Manduca sexta. Proc. Biol. Sci. 274, 977-981.

Konopova, B. and Jindra, M. 2007. Juvenile hormone resistance gene Methoprene-tolerant controls entry into metamorphosis in the beetle Tribolium castaneum. Proc. Natl. Acad. Sci. USA 104, 10488-10493.

Konopova, B., Smykal, V. and Jindra, M. 2011. Common and distinct roles of juvenile hormone signaling genes in metamorphosis of holometabolous and hemimetabolous insects. PLoS One 6, e28728.

Li, K.L., Yuan, S.Y., Nanda, S., Wang, W.X., Lai, F.X., Fu, Q. and Wan, P.J. 2018. The Roles of E93 and Kr-h1 in Metamorphosis of Nilaparvata lugens. Front. Physiol. 9, 1677.

Lo, P.C., Frasch, M., 1999. Sequence and expression of myoglianin, a novel Drosophila gene of the TGF-beta superfamily. Mech. Dev. 86, 171-175.

Lozano, J. and Belles, X. 2011. Conserved repressive function of Kruppel homolog 1 on insect metamorphosis in hemimetabolous and holometabolous species. Sci. Rep. 1, 163.

McPherron, A.C., Lawler, A.M. and Lee, S.J. 1997. Regulation of skeletal muscle mass in mice by a new TGF-beta superfamily member. Nature 387, 83-90.

Minakuchi, C., Namiki, T. and Shinoda, T. 2009. Kruppel homolog 1, an early juvenile hormone-response gene downstream of Methoprene-tolerant, mediates its antimetamorphic action in the red flour beetle Tribolium castaneum. Dev. Biol. 325, 341-350.

Minakuchi, C., Namiki, T., Yoshiyama, M. and Shinoda, T. 2008. RNAi-mediated knockdown of juvenile hormone acid O-methyltransferase gene causes precocious metamorphosis in the red flour beetle Tribolium castaneum. FEBS J. 275, 2919-2931.

Moss-Taylor, L., Upadhyay, A., Pan, X., Kim, M.-J. and O'Connor, M.B. 2019. Motoneuronderived Activin $\beta$ regulates Drosophila body size and tissue-scaling during larval growth and adult development. Genetics 213, 1447-1464.

Nagamine, K., Ishikawa, Y. and Hoshizaki, S. 2016. Insights into how longicorn beetle larvae determine the timing of metamorphosis: starvation-induced mechanism revisited. PLoS One $11, \mathrm{e} 0158831$.

Namigai, E.K.O. and Suzuki, Y. 2012. Functional conservation and divergence of BMP ligands in limb development and lipid homeostasis of holometabolous insects. Evol. Dev. 14, 296-310. 
Nijhout, H.F., 1975. Threshold size for metamorphosis in tobacco hornworm, Manduca-sexta (L). Biol. Bull. 149, 214-225.

Ohtaki, T., Takeuchi, S. and Mori, K. 1971. Juvenile hormone and synthetic analogues: effects on larval moult of silkworm, Bombyx mori. Jpn J Med Sci Biol 24, 251-255.

Riddiford, L.M. 1996. Juvenile hormone: The status of its "status quo" action. Arch. Insect Biochem. Physiol. 32, 271-286.

Riddiford, L.M. and Ashburner, M. 1991. Effects of juvenile hormone mimics on larval development and metamorphosis of Drosophila melanogaster. Gen. Comp. Endocrinol. $82,172-183$.

Santos, C.G., Fernandez-Nicolas, A. and Belles, X. 2016. Smads and insect hemimetabolan metamorphosis. Dev. Biol. 417, 104-113.

Shafiei, M., Moczek, A.P. and Nijhout, H.F. 2001. Food availability controls the onset of metamorphosis in the dung beetle Onthophagus taurus (Coleoptera: Scarabaeidae). Phys. Entomol. 26, 173-180.

Smykal, V., Daimon, T., Kayukawa, T., Takaki, K., Shinoda, T. and Jindra, M. 2014. Importance of juvenile hormone signaling arises with competence of insect larvae to metamorphose. Dev. Biol. 390, 221-230.

Suzuki, Y., Koyama, T., Hiruma, K., Riddiford, L.M. and Truman, J.W. 2013. A molt timer is involved in the metamorphic molt in Manduca sexta larvae. Proc. Natl. Acad. Sci. USA $110,12518-12525$.

Tan, A., Tanaka, H., Tamura, T., Shiotsuki, T., 2005. Precocious metamorphosis in transgenic silkworms overexpressing juvenile hormone esterase. Proc. Natl. Acad. Sci. USA 102, 11751-11756.

Terao, M., Hirose, Y. and Shintani, Y. 2015. Food-availability dependent premature metamorphosis in the bean blister beetle Epicauta gorhami (Coleoptera: Meloidae), a hypermetamorphic insect that feeds on grasshopper eggs in the larval stage. Entomological Science 18, 85-93.

Tomoyasu, Y. and Denell, R.E. 2004. Larval RNAi in Tribolium (Coleoptera) for analyzing adult development. Dev. Genes Evol. 214, 575-578.

Whittemore, L.A., Song, K., Li, X., Aghajanian, J., Davies, M., Girgenrath, S., Hill, J.J., Jalenak, M., Kelley, P., Knight, A., Maylor, R., O'Hara, D., Pearson, A., Quazi, A., Ryerson, S., 
bioRxiv preprint doi: https://doi.org/10.1101/2020.04.21.053835; this version posted April 23, 2020. The copyright holder for this preprint (which

was not certified by peer review) is the author/funder, who has granted bioRxiv a license to display the preprint in perpetuity. It is made available under aCC-BY-NC-ND 4.0 International license.

Tan, X.Y., Tomkinson, K.N., Veldman, G.M., Widom, A., Wright, J.F., Wudyka, S., Zhao, L. and Wolfman, N.M. 2003. Inhibition of myostatin in adult mice increases skeletal muscle mass and strength. Biochem. Biophys. Res. Commun. 300, 965-971. 


\section{FIGURE LEGENDS}

Figure 1. Nutritional conditions affect threshold size. (A) A plot of mass at the end of the fourth instar vs developmental fate. (Top) Larvae fed a 40\% diet during the third instar. (Middle) Larvae starved during the third instar starting day 1. (Bottom) Larvae fed a $40 \%$ diet throughout the larval instar. Red line indicates the threshold size for larvae fed a $40 \%$ diet throughout much of their larval life. "Supernumerary larval molt" indicates that the fifth instar larvae molted into another larval instar. "Last instar" indicates that the larvae initiated wandering at the end of the fifth instar. Larvae were tracked until they exhibted signs of pupation or initiated a supernumerary molt. (B) Experimental scheme. (C) The average masses of five larvae at the end of the fourth instar were plotted against the percentage of larvae that entered final larval instar. Solid black line represents larvae that were starved during the third instar. Solid gray line represents larvae that were fed a $40 \%$ diet during the third instar. Dotted line represents larvae that were fed a $40 \%$ diet during the majority of the growth period. Triangles indicate the mass where $50 \%$ of larvae entered the final instar (i.e. threshold size). Lines represent Gompertz 3P model fits. Blue line represents threshold size of hypoxia-treated larvae.

Figure 2. Third and fourth instar hypoxia-treated larvae have similar threshold sizes whereas $40 \%$ diet delays the attainment of threshold size. (A) A plot of mass at the end of the fourth instar vs developmental fate. Red line indicates the threshold size for the hypoxia-treated larvae. Blue line indicates the threshold size for the $40 \%$ diet fed larvae. "Supernumerary larval molt" indicates that the fifth instar larvae molt into another larval instar. "Last instar" indicates that the larvae initiated wandering at the end of the fifth instar. (B) Scheme of experimental treatments. Lines represent duration of treatments. Larvae were subjected to hypoxic conditions for the duration of the third or fourth instar, or fed a $40 \%$ diet starting in early larval life, and tracked until they exhibited signs of wandering or initiated a supernumerary molt. (C) The moving average masses of five larvae at the end of the fourth instar were plotted against the percentage of larvae that entered final larval instar. Solid black line represents larvae that were placed in hypoxic condition during the third instar. Solid gray line represents larvae that were placed in hypoxic 
31 the majority of the growth period. Triangles indicate the mass where $50 \%$ of larvae entered the

32 final instar (i.e. the threshold size). Lines represent Gompertz 3P model fits.

Figure 3. The relative dry mass of muscles/integuments is reduced in larvae fed a $40 \%$ diet relative to hypoxia-treated larvae. (A) Dry mass of muscles/integuments plotted against wet mass of fourth instar larvae. (B) Dry mass of fat body plotted against wet mass of fourth instar larvae. Red lines represent the relationship between the threshold sizes (triangles) and muscle/integument mass or fat body mass at the threshold sizes. Larvae were either placed in hypoxic conditions during the third instar or reared on a $40 \%$ diet throughout much of the larval stage. All dissections were performed at the end of the fourth instar. The dotted confidence ellipses are drawn at the $95 \%$ confidence level.

Figure 4. Expression of myo in normoxia/normal diet-fed M. sexta larvae. (A) Myo expression in the anterior half of the whole body was determined at HCS of the second, third and fourth instar of untreated larvae. Error bars represent standard error. Expression represents mean of three biological replicates, each with three technical replicates. (B-D) Myo expression in the muscles (B), fat body (C) and CNS (D) of normoxia/normal diet-fed third and fourth instar larvae. Error bars represent standard error. Expression represents mean of three or four biological replicates, each with three technical replicates. * indicates a statistically significant difference

50 (Student's t-test: $\mathrm{p}<0.05$ ).

Figure 5. myo expression in muscles is signficantly elevated in post-threshold size larvae of both hypoxia-treated larvae and larvae fed a $\mathbf{4 0 \%}$ diet. (A) myo expression in muscles, fat

54 body and the CNS of hypoxia-treated larvae. Larvae were reared under hypoxa conditions during 55 the third instar. (B) myo expression in muscles, fat body and the CNS of larvae fed a $40 \%$ diet.

56 (C) myo expression in muscles and fat body of hypoxia-treated and 40\% diet-fed larvae weighing

$57 \quad 0.7 \mathrm{~g}$ to $0.8 \mathrm{~g}$. At this weight range, hypoxia-treated larvae are above the threshold size whereas

$5840 \%$ diet-fed larvae are below the threshold size. For all samples, larvae were dissected at the

59 end of the fourth instar when the larvae began to initiate a molt. Expression represents mean of

60 4-5 biological replicates. Each biological replicate was run with three technical replicates.

61 Student's t-test: * denotes $\mathrm{p}<0.05 ; * *$ denotes $\mathrm{p}<0.01 ; * * *$ denotes $\mathrm{p}<0.005$. 
63 Figure 6. myo is upregulated in post-threshold size $T$. castaneum larvae. (A) Determination

64 of threshold size. T. castaneum undergo variable number of molts passing through at least seven instars. On day 1 of the final instar, T. castaneum larvae undergo pupal commitment (Kamsoi and Belles, 2019). If larvae undergo pupation at the end of the instar, the larval weight on day 1 must be the threshold size. The moving average masses of five day 1 seventh instar larvae were plotted against the percentage of larvae that pupated. Our data show that at $2.1 \mathrm{mg}, 50 \%$ of larvae have reached the pupal commitment point, indicating that if seventh instar larvae have reached $2.1 \mathrm{mg}$ at the time of the molt, they must have reached the threshold size. (B) Determination of myo in freshly molted sixth instar (below the threshold size) and seventh instar

72 larvae above $2.1 \mathrm{mg}$ (above the threshold size). Expression represents the whole body minus the gut and fat body. ${ }^{*}$ denotes statistically significant difference (Student's t-test: $\mathrm{p}<0.05$ ).

Figure 7. Knockdown of myo results in molting delays and prolonged larval stage of $\boldsymbol{T}$. castaneum. (A) Knockdown verification showing that myo dsRNA injection leads to reduced expression of myo in myo dsRNA-injected larvae relative to $a m p^{r}$ dsRNA-injected larvae. Cycle number used in gel image: $m y o=35$ cycles; $r p 49=25$ cycles. (B) Duration of each larval instar post dsRNA injection. Bars represent the average duration of each larval instar. Averages were combined for larvae that pupated after the seventh and eighth instar because the duration of each instar did not differ between the groups. $a m p^{r}$ dsRNA-injected larvae were used as the control. Error bars represent standard error. (C) Duration of larval period in $a m p^{r}$ and myo dsRNAinjected larvae. Three of the myo dsRNA-injected larvae were still alive after 215 days ( 7 months). Day 0 sixth instar larvae were injected with $0.25 \mu \mathrm{L}$ of dsRNA using a $10 \mu \mathrm{L}$ glass capillary needle. (D) Growth trajectory of myo and $a m p^{r}$ dsRNA-injected larvae. Lines represents average larval masses for each time point: black lines represent $a m p^{r}$ dsRNA-injected larvae that underwent two larval molts post dsRNA injections prior to metamorphosis; dotted lines represent $a m p^{r}$ dsRNA-injected larvae that underwent one larval molt post dsRNA injections prior to metamorphosis; gray lines represent $v v l$ dsRNA-injected larvae. Filled circles represent $a m p^{r}$ dsRNA-injected larvae; "X” represents prepupal masses of $a m p^{r}$ dsRNA-injected larvae; open circles represent myo dsRNA-injected larvae. (E) Fate of larvae at different masses. Larvae were weighed one day after a molt. Filled circles represent $a m p^{r}$ dsRNA-injected larvae; open circles 
93 represent myo dsRNA-injected larvae. Each larva may be represented by several circles if they

94 continued to undergo multiple supernumerary molts.

95

96 Figure 8. Hypothetical model for how growth of the body is coupled to initiation of

97 metamorphosis. In organisms undergoing determinate growth, the growth phase and the

98 reproductive phases are temporally separated. In this model, the growing tissues (primarily

99 muscles) produce increasing amounts of Myo. Once a threshold level is reached, Myo triggers

100 the end of the growth phase by affecting the neuroendocrine regulators of metamorphosis.

101 Because the same factor acts as both an indicator of growth and initiator of metamorphosis,

102 metamorphosis can be triggered precisely at the threshold size. Dotted line indicates potential

103 positive autocrine feedback.

104

105 


\section{Figure 1}

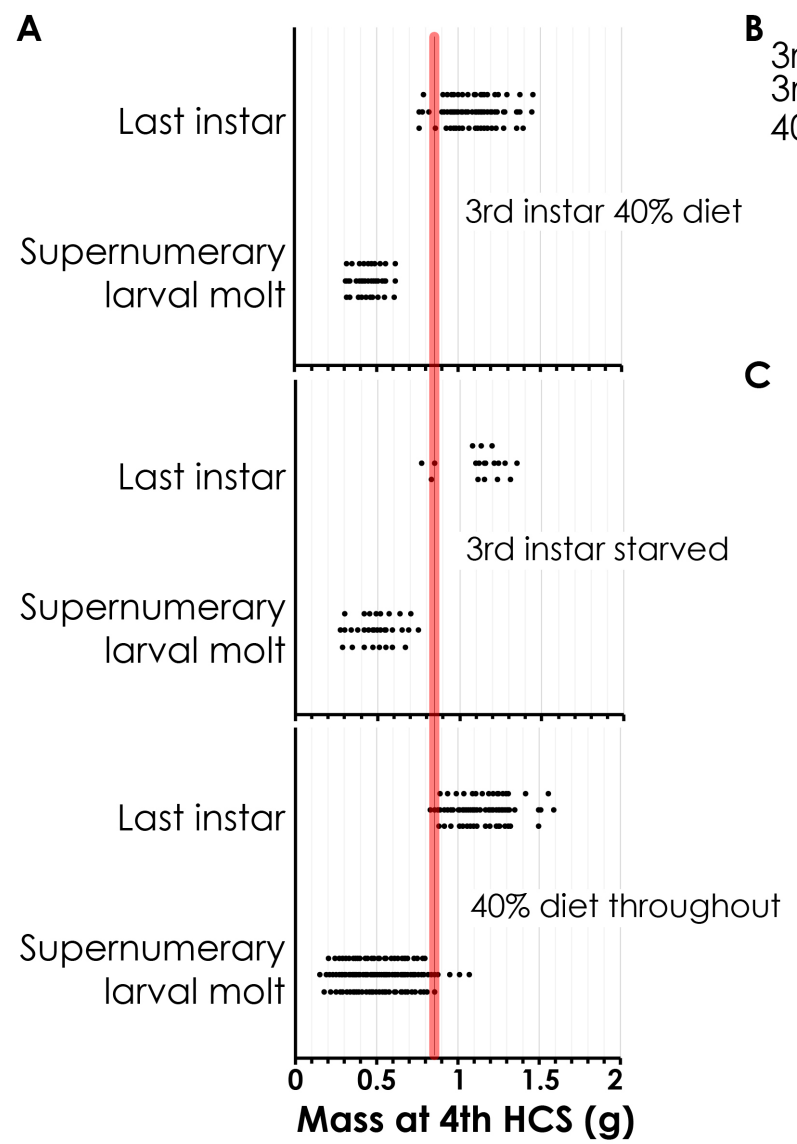

A

Mass at 4th HCS (g) 3rd starved 3 rd $40 \%$ diet $40 \%$ diet throughout Instar

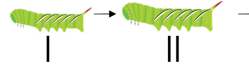

III IV
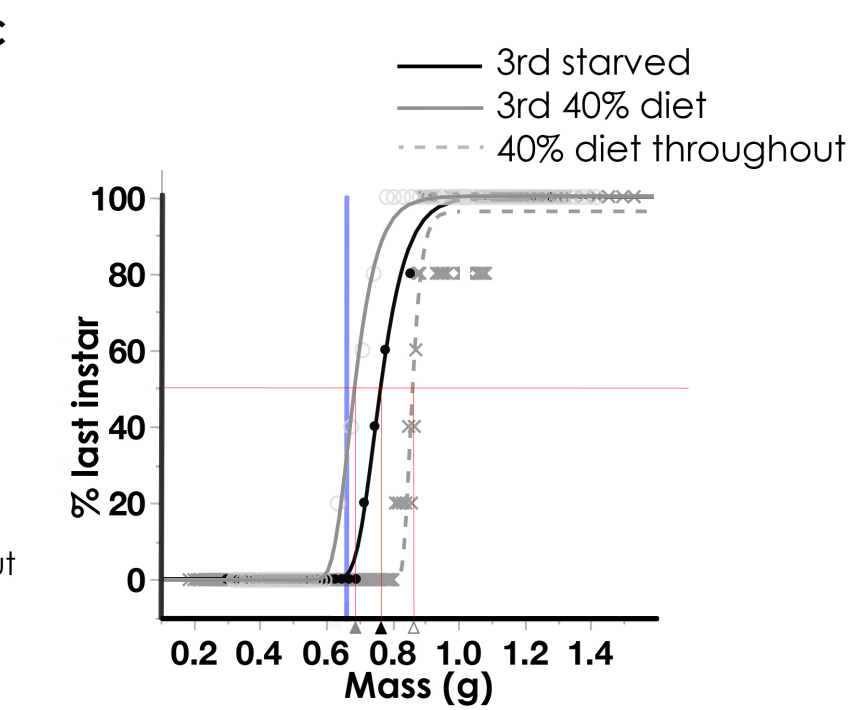


\section{Figure 2}

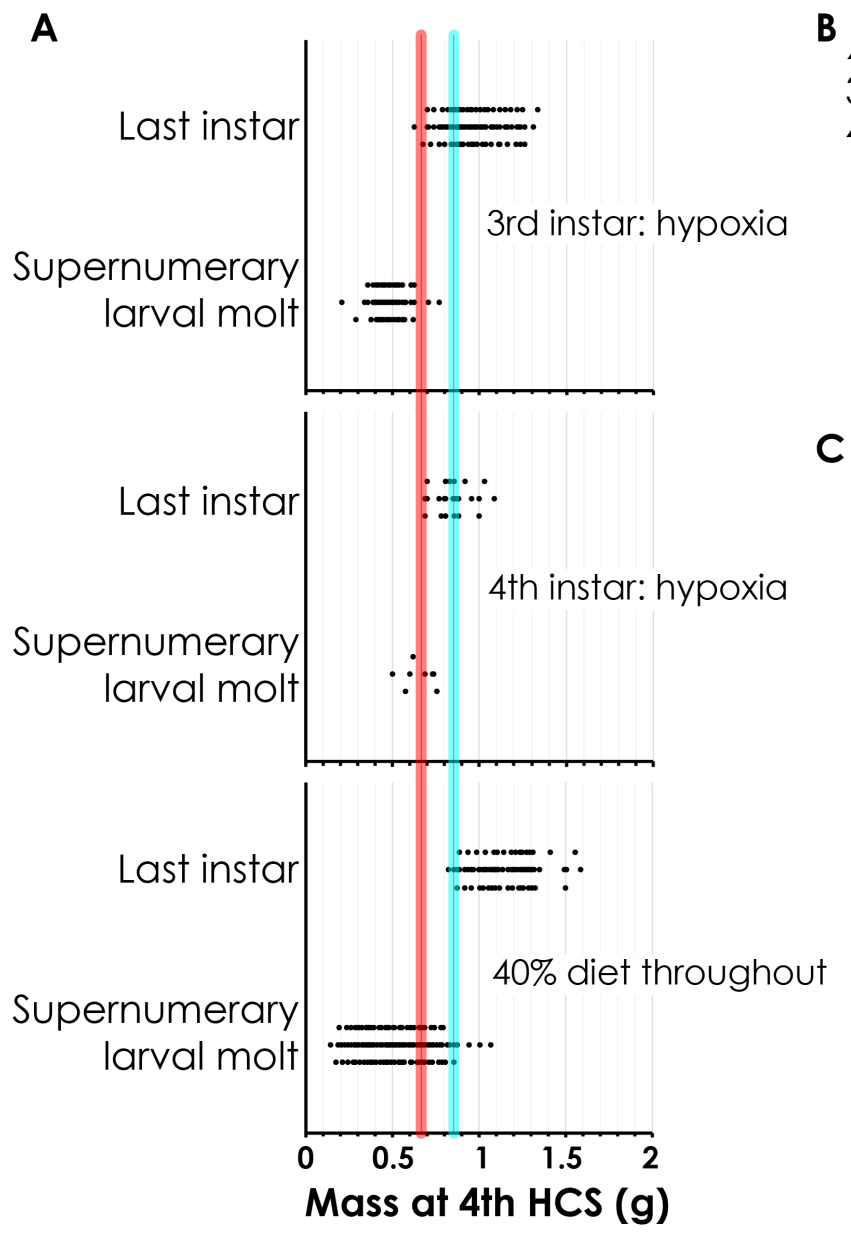

B th hypoxia

3rd hypoxia

$40 \%$ diet

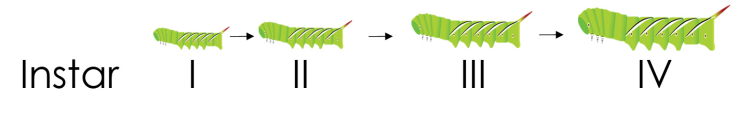

C
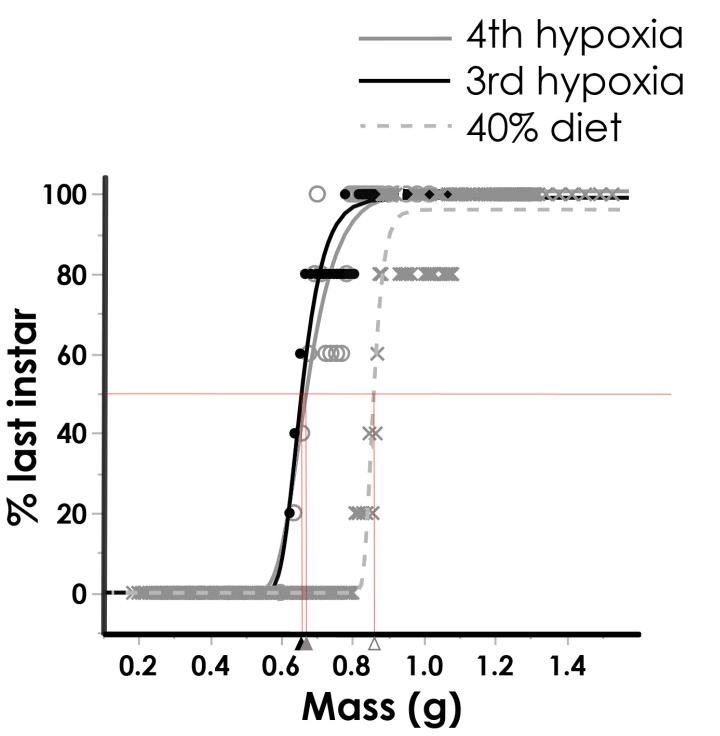


\section{Figure 3}
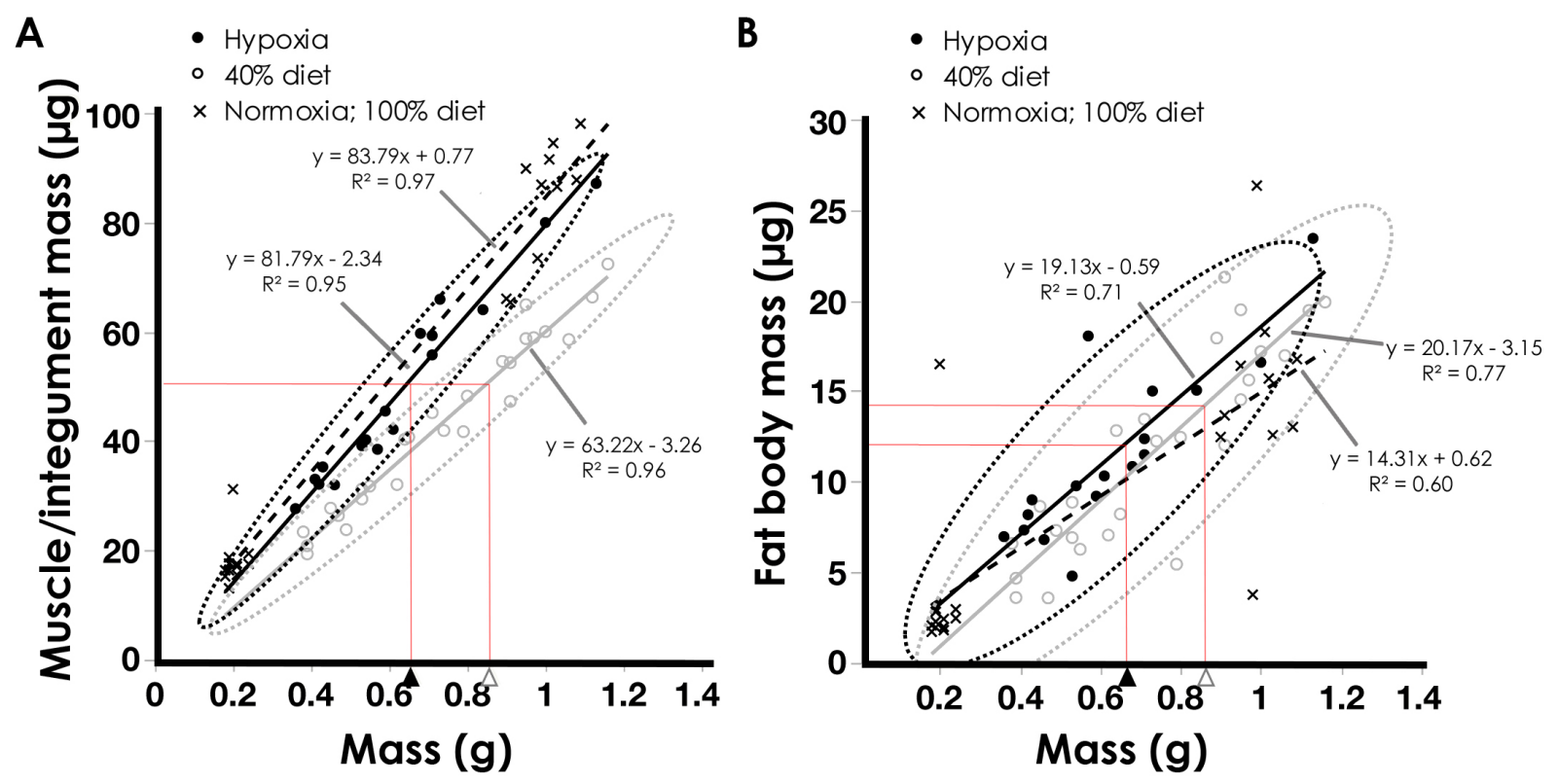
Figure 4

A

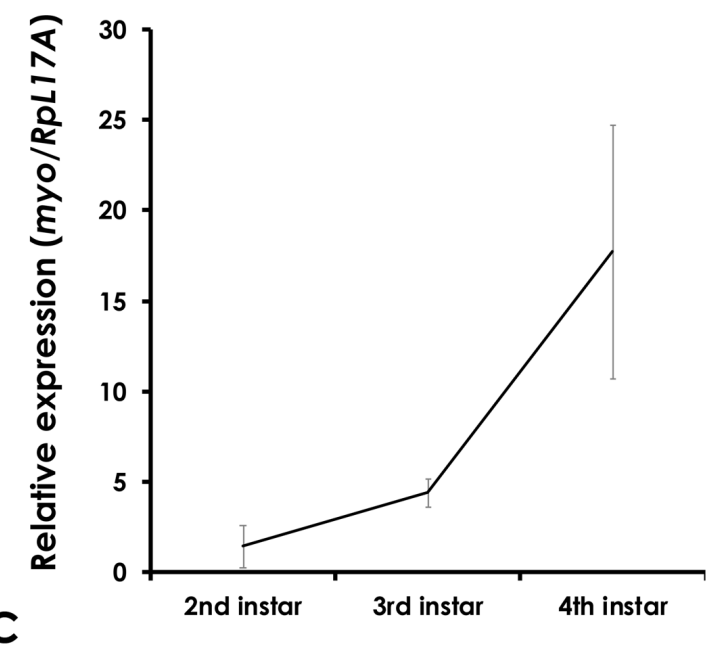

B

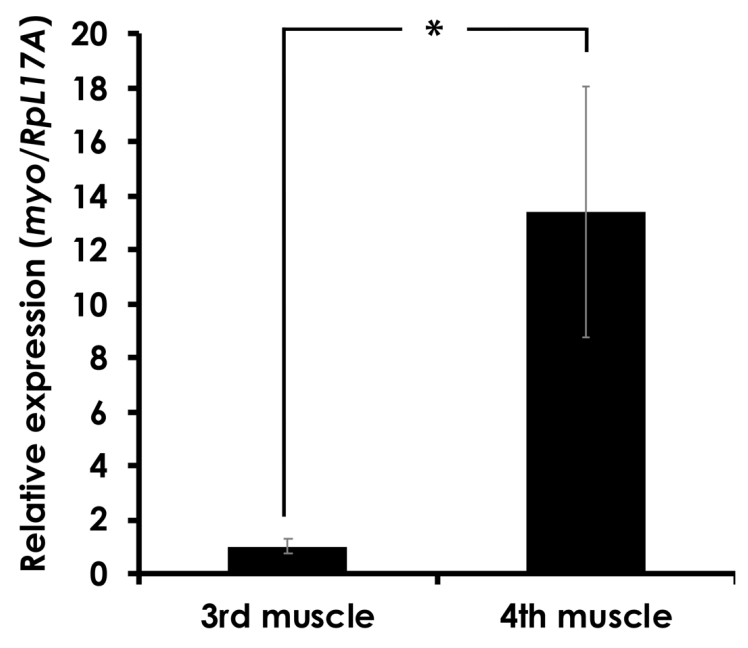

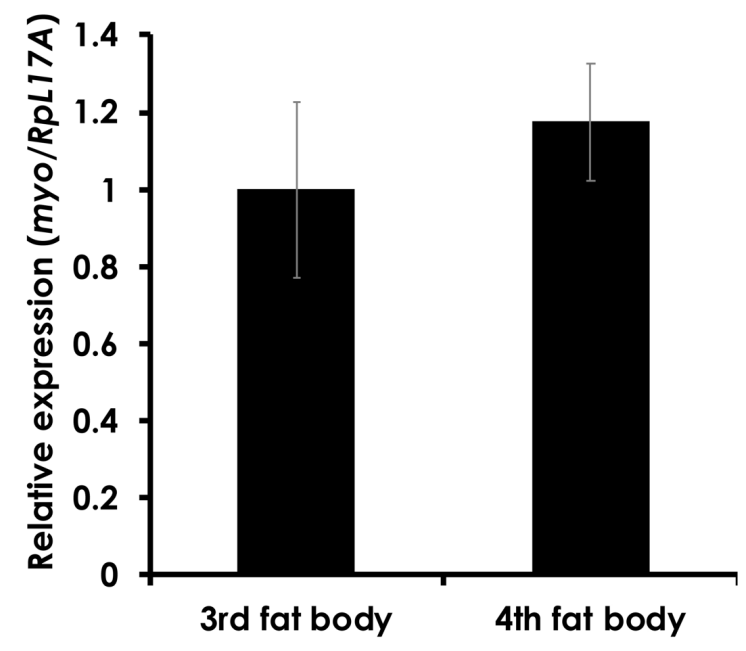

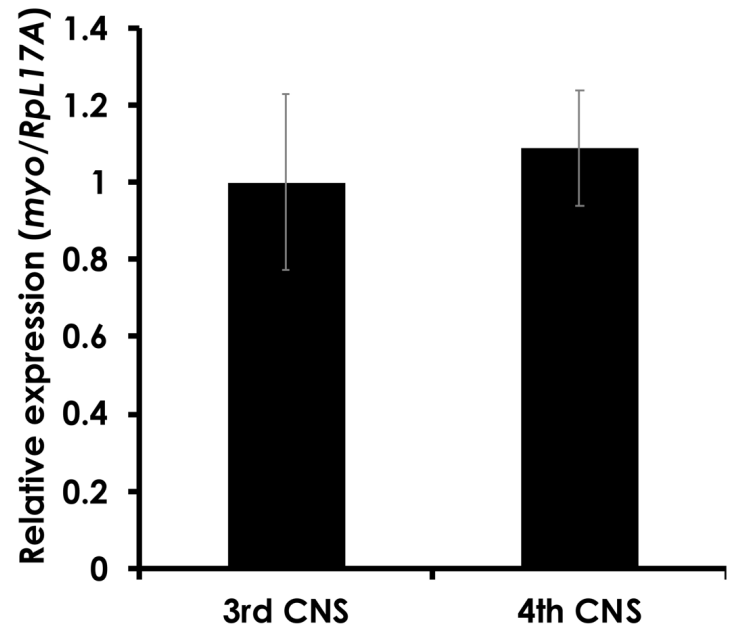




\section{A. Hypoxia-treated larvae}

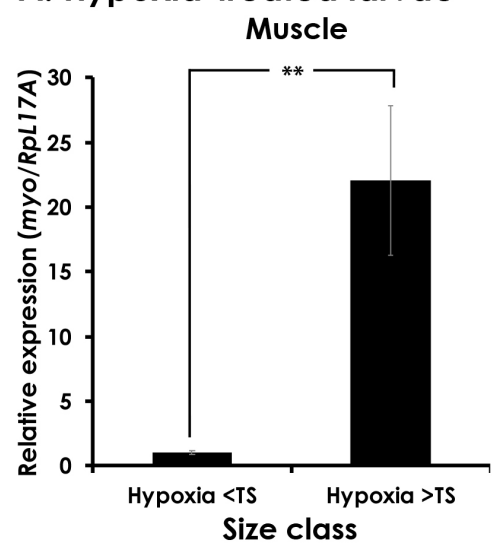

\section{B. $40 \%$ diet-fed larvae}

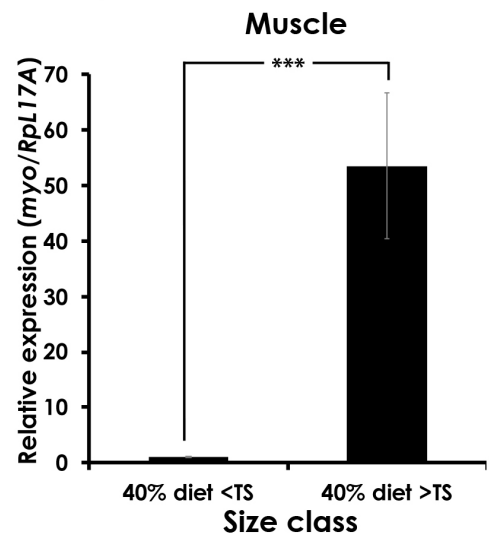

Fat body

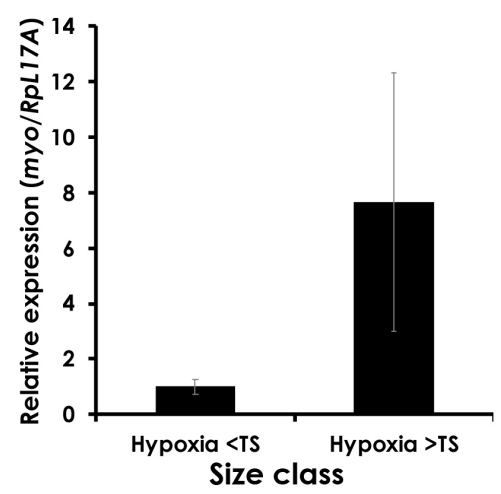

Fat body

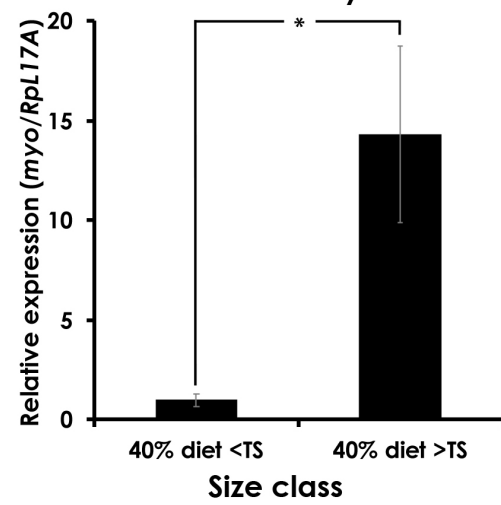

CNS

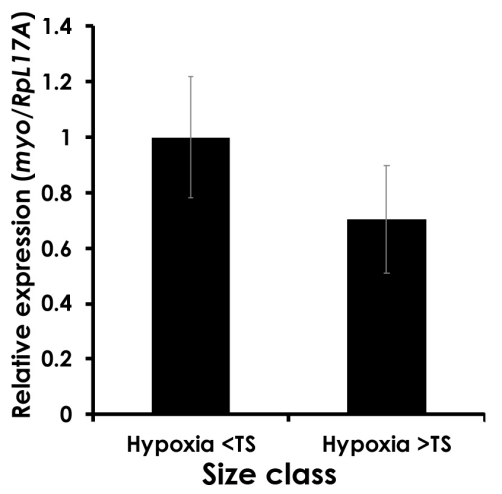

CNS

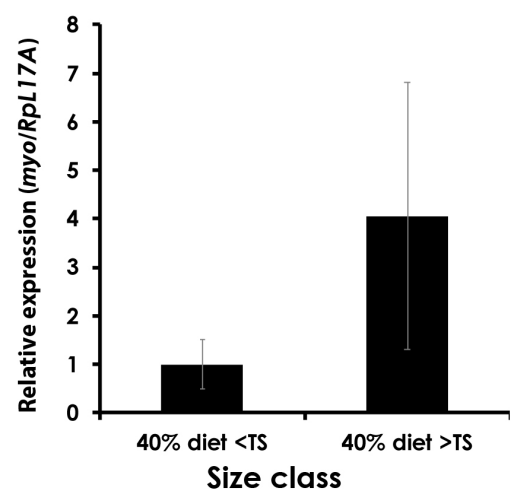

\section{Hypoxia-treated and $40 \%$ diet fed larvae at $0.7 \mathrm{~g}-0.8 \mathrm{~g}$}
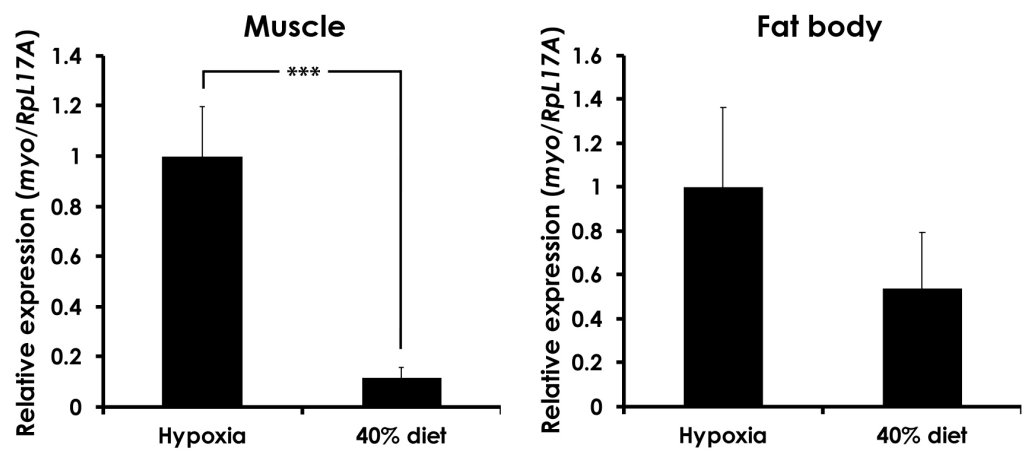


\section{Figure 6}
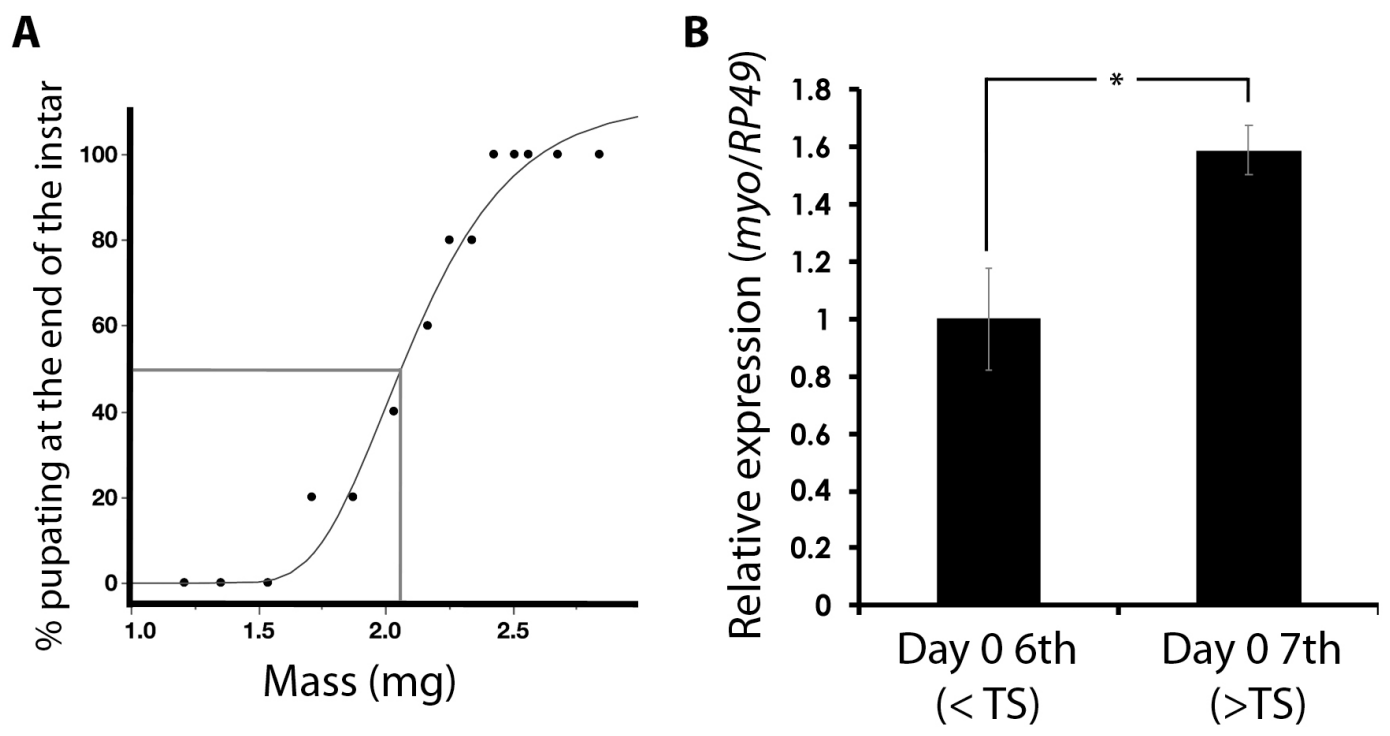
Figure 7

A

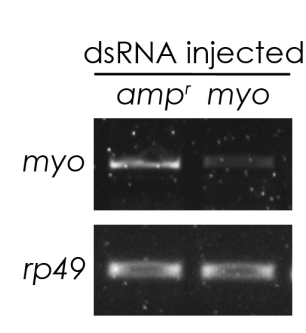

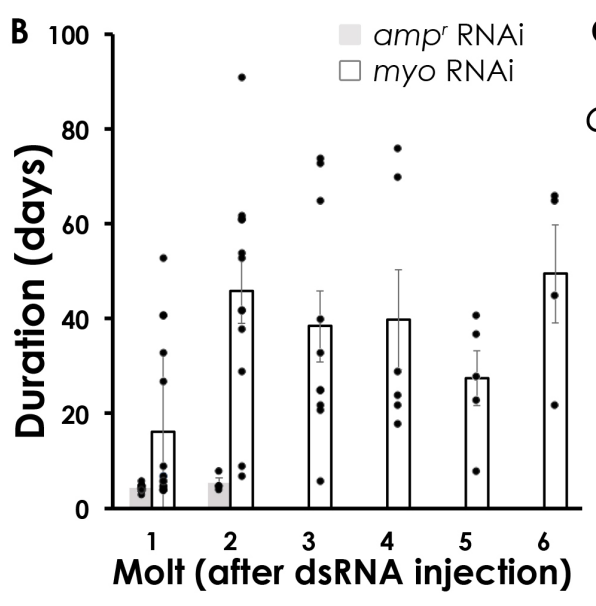

C

myo 0000000000 D 0

E

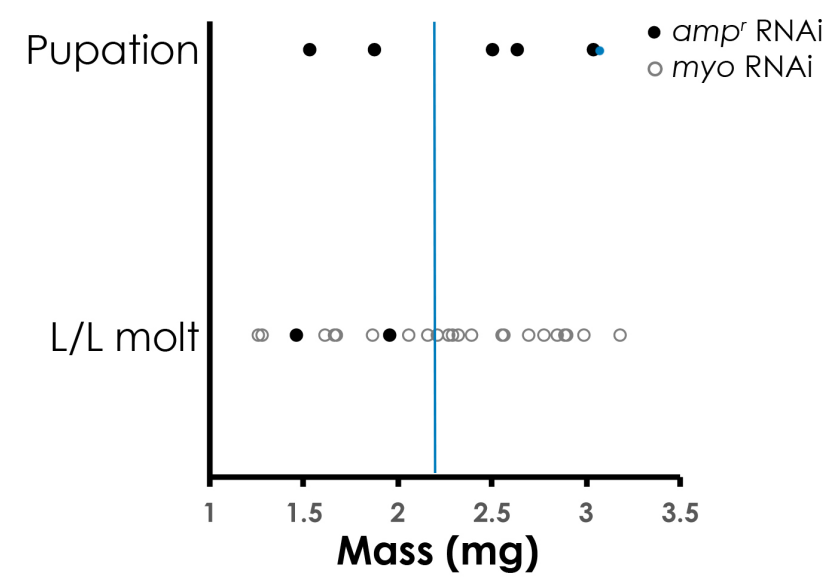


bioRxiv preprint doi: https://doi.org/10.1101/2020.04.21.053835; this version posted April 23, 2020. The copyright holder for this preprint (which was not certified by peer review) is the author/funder, who has granted bioRxiv a license to display the preprint in perpetuity. It is made available under aCC-BY-NC-ND 4.0 International license.

Figure 8

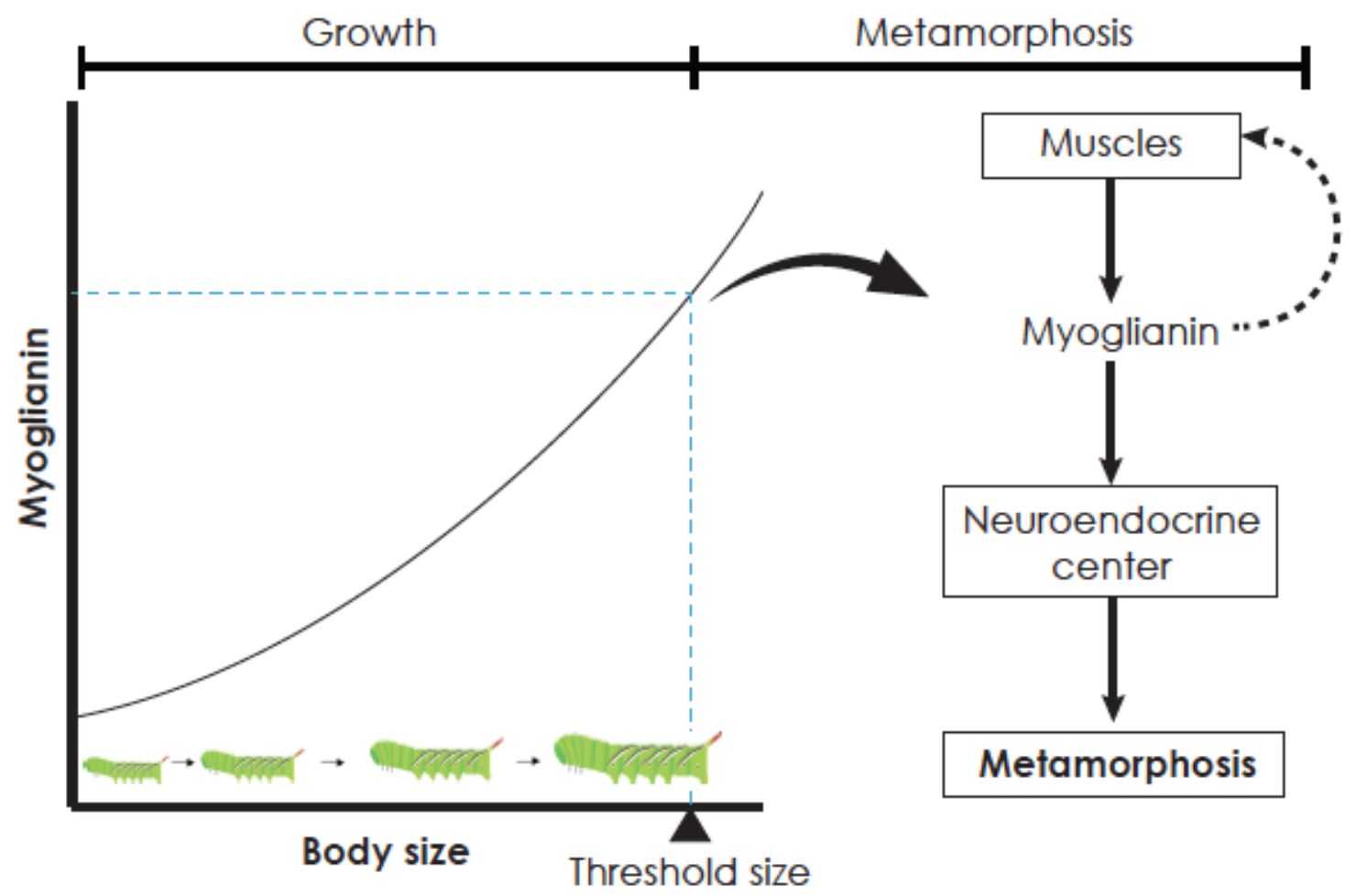


106 Table 1. Summary of molting events in myo knockdown larvae. Sixth and seventh instar

107 larvae were injected with myo or $a m p^{r}$ dsRNA. Injected animals were checked daily for molts,

108 prepupal formation and death.

\begin{tabular}{|c|c|c|c|c|c|c|c|c|}
\hline \multirow[b]{2}{*}{ dsRNA } & \multirow[b]{2}{*}{$\begin{array}{c}\text { Stage } \\
\text { injected }\end{array}$} & \multirow[b]{2}{*}{$\begin{array}{c}\# \\
\text { injected }\end{array}$} & \multicolumn{3}{|c|}{ Pupating larvae } & \multicolumn{3}{|c|}{$\begin{array}{l}\text { Larvae that never pupated } \\
\text { and died as larvae }\end{array}$} \\
\hline & & & $\begin{array}{c}\text { \# pupating } \\
\text { without } \\
\text { molting }\end{array}$ & $\begin{array}{c}\text { \# molted } \\
\text { once } \\
\text { before } \\
\text { pupation }\end{array}$ & $\begin{array}{c}\text { \# molted } \\
\text { twice } \\
\text { before } \\
\text { pupation }\end{array}$ & $\begin{array}{l}\text { \# never } \\
\text { molted }\end{array}$ & $\begin{array}{l}\text { \# molted } \\
\text { once }\end{array}$ & $\begin{array}{c}\text { \# molted } \\
\text { more } \\
\text { than } \\
\text { twice }\end{array}$ \\
\hline \multirow[t]{2}{*}{$a m p^{r}$} & Day $06^{\text {th }}$ & 18 & - & 11 & 4 & - & 3 & - \\
\hline & Day $07^{\text {th }}$ & 7 & 7 & - & - & & & - \\
\hline \multirow[t]{2}{*}{ myo } & Day $06^{\text {th }}$ & 18 & - & - & - & 3 & 4 & 11 \\
\hline & Day $07^{\text {th }}$ & 6 & - & - & - & & 4 & 2 \\
\hline
\end{tabular}




\section{SUPPORTING INFORMATION}

A

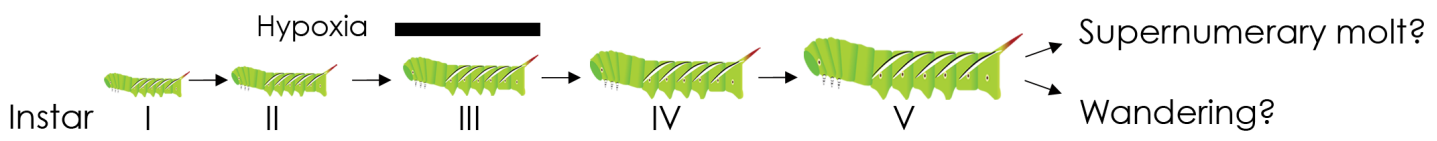

B

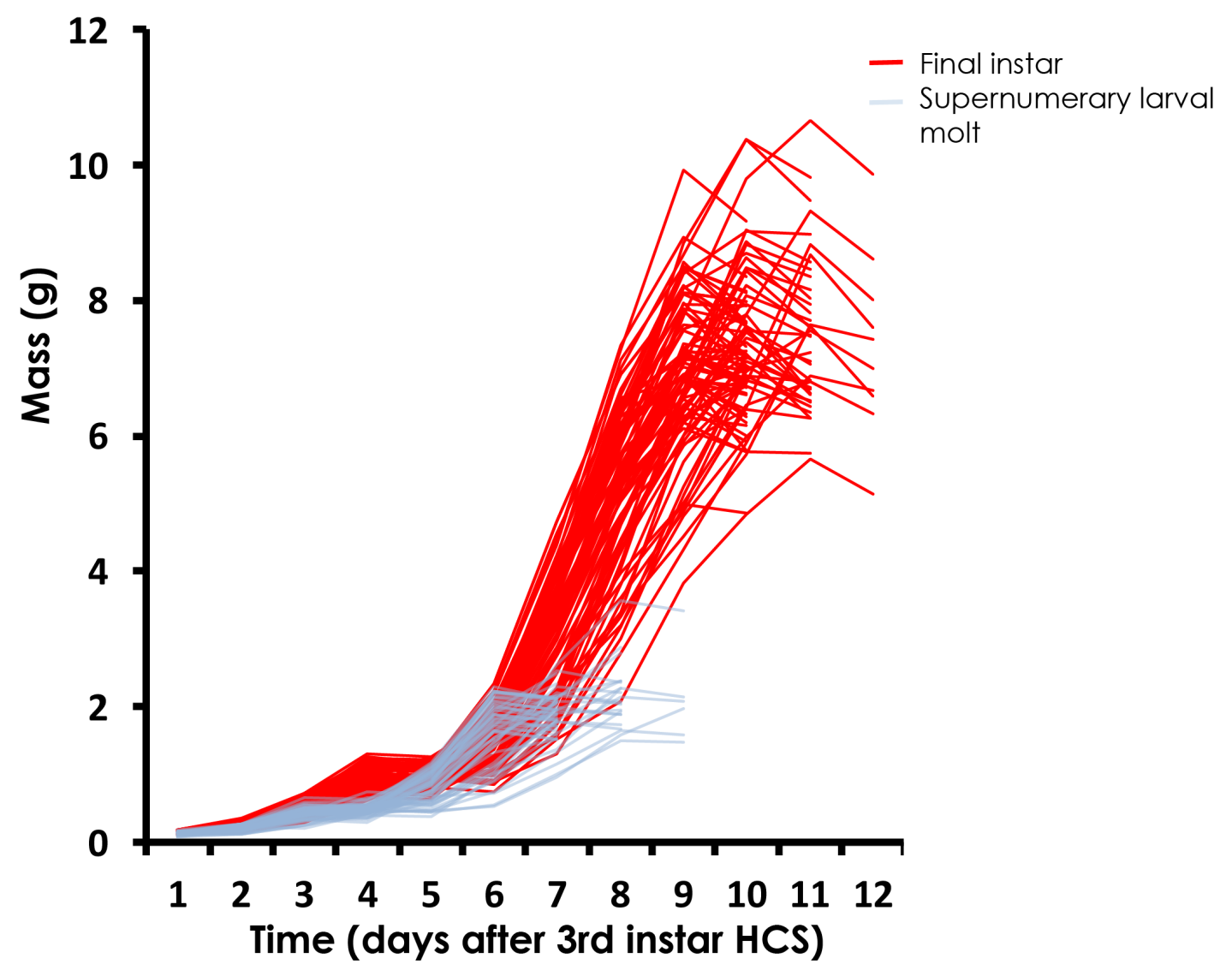

Supplemental figure S1. Hypoxia generates two developmental trajectories for

Manduca sexta. (A) A scheme showing the timing of hypoxia treatment. (B) Individual growth trajectories of larvae that underwent wandering (red) or supernumerary molt at the end of the fifth instar (light blue). Larvae were subjected to hypoxic conditions from the beginning to the end of the third instar. Masses were recorded at the end of the third instar and every day after. 


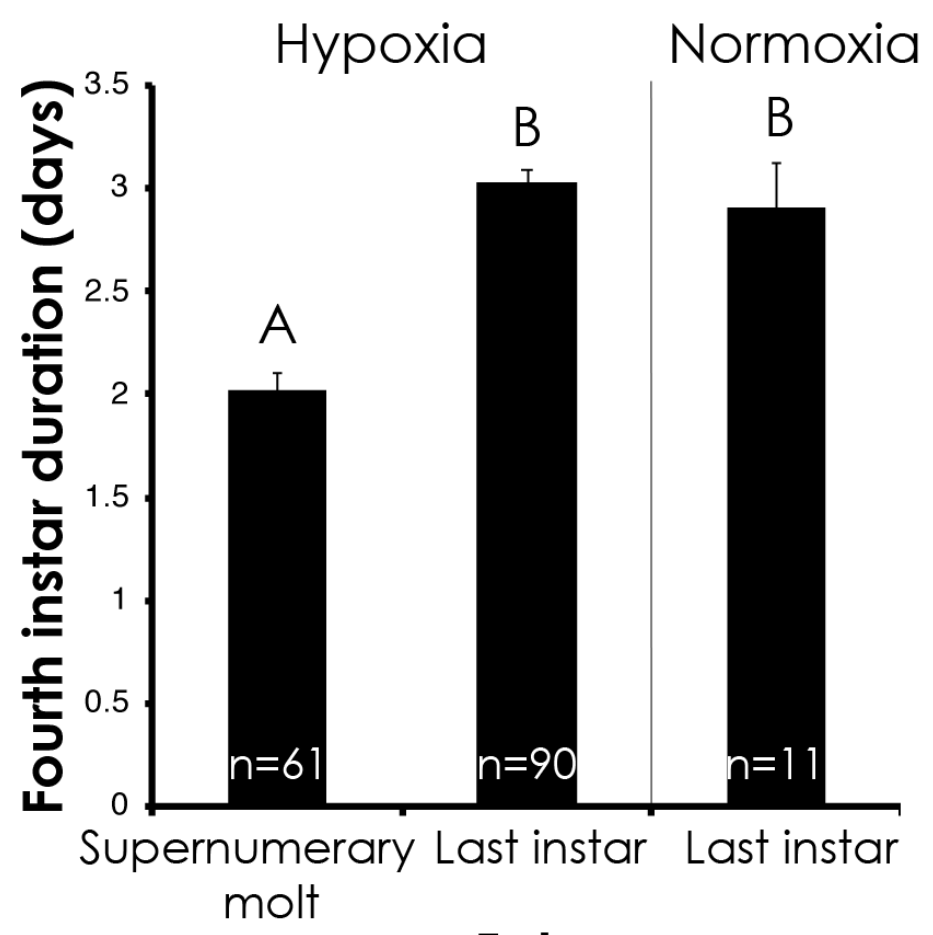

Fate

Supplemental figure S2. Fourth instar duration predicts developmental fate of the larva. Fourth instar duration (mean \pm SE) of larvae that wandered at the end of the fifth instar (denoted "last instar" and those that underwent a supernumerary molt (denoted "supernumerary larval molt") are shown for larvae that were reared under hypoxic or normoxic conditions during the third instar. Days were counted from day 0 of the fourth instar. One-way ANOVA: $F(2,159)=125.556, \mathrm{p}<0.0001$. Means not sharing the same letter are statistically significant (Tukey HSD, $\mathrm{p}<0.0001$ ). 
A

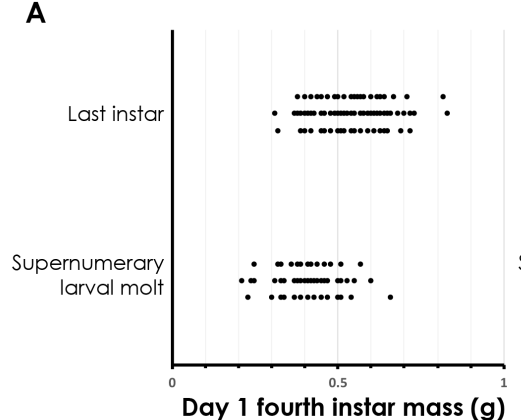

B

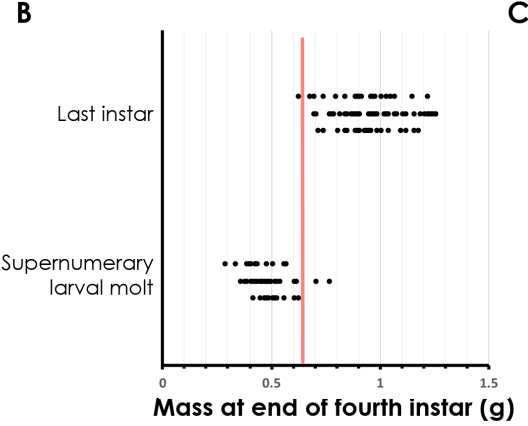

C

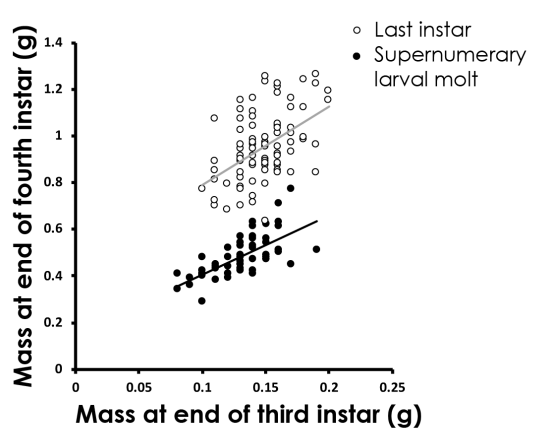

Supporting figure S3. Mass at the end of the fourth instar predicts the

developmental fates of larvae. (A) The mass at first day of the fourth instar is a poor predictor of nature of subsequent molt. "Supernumerary larval molt" denotes fifth instar larvae that molted into another larval instar. "Last instar" denotes fifth instar that initiated wandering. (B) The decision to enter supernumerary stage is made at the end of the fourth instar. Red line indicates the estimated threshold size. (C) A plot of mass at the end of the third instar vs mass at the end of the fourth instar, showing that the mass at the end of the third instar is a poor predictor of threshold size. Filled circles are larvae that underwent a supernumerary larval molt; open circles denote larvae that wandered at the end of the fifth instar. All larvae were subjected to hypoxic conditions during the third instar and then tracked for supernumerary molt/wandering. 


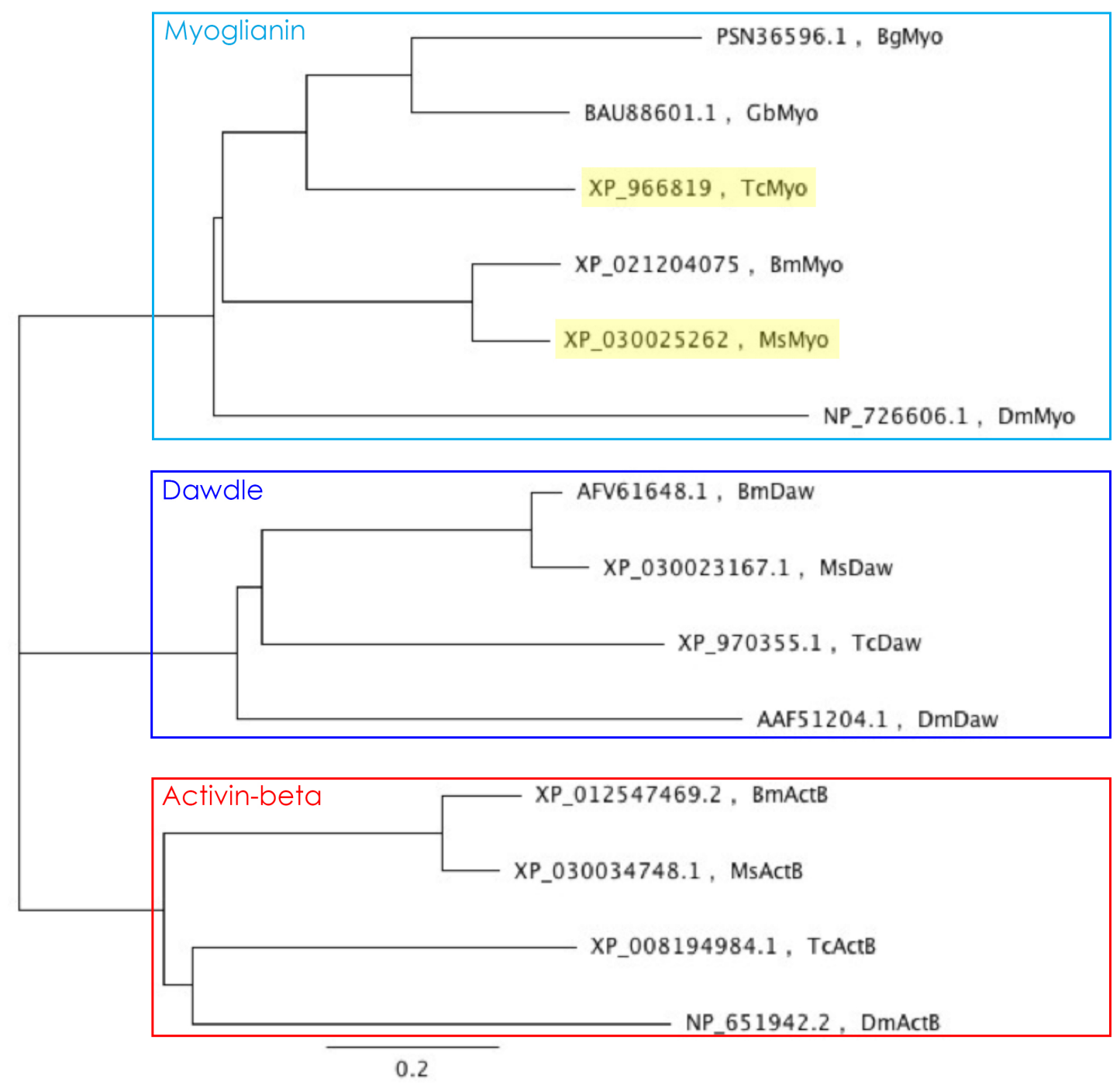

Supplemental figure S4. Phylogenetic tree of the three Activin ligands, Activin-beta (ActB), Dawdle (Daw) and Myoglianin (Myo) predicted from genome sequences of Drosophila melanogaster (Dm), Blattella germanica $(B g)$, Gryllus bimaculatus $(\mathrm{Gb})$, Tribolium castaenum (Tc), Bombyx mori (Bm) and Manduca sexta (Ms). 

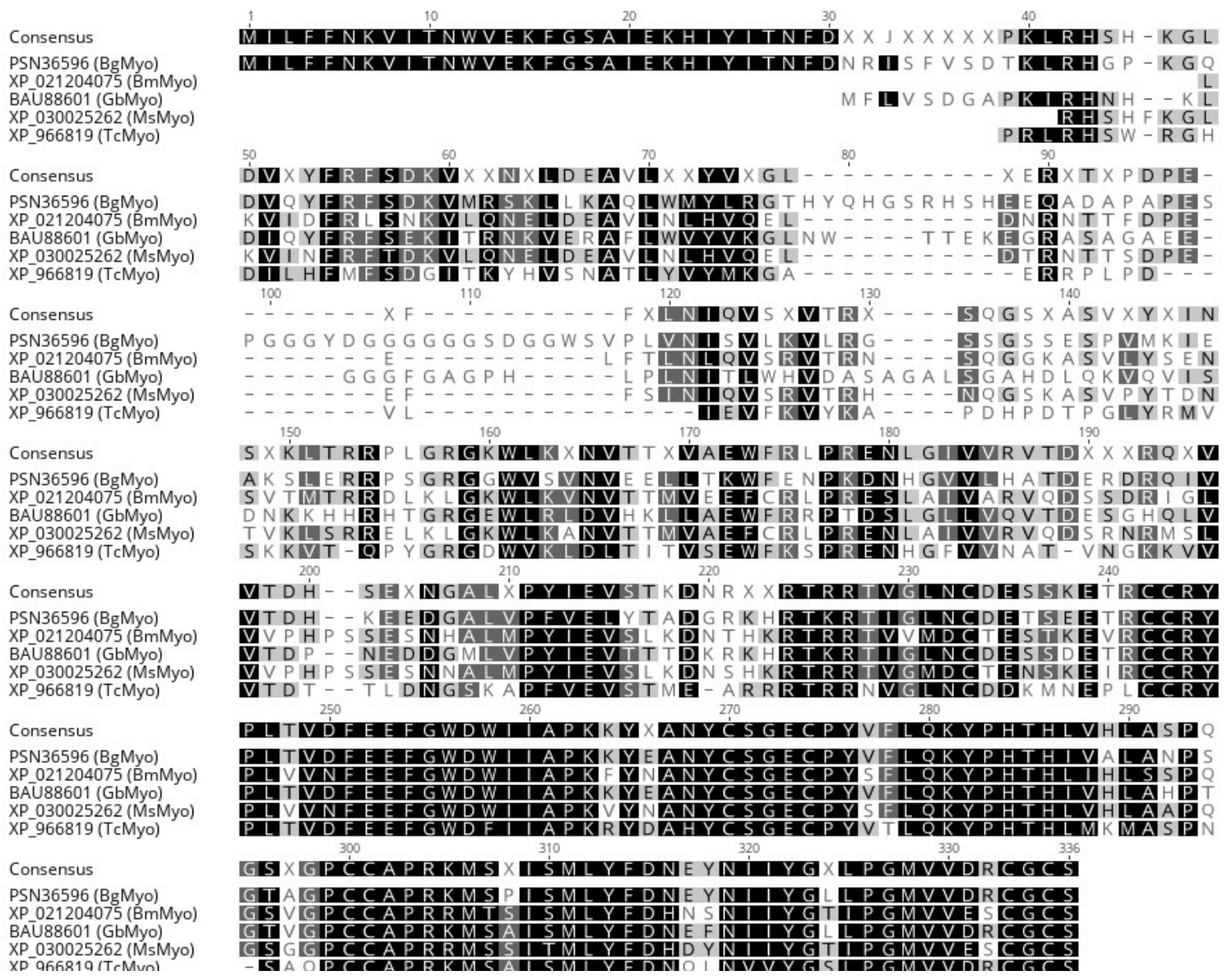

Supplemental figure S5. Amino acid alignment of Myoglianin from Blattella germanica (Bg), Bombyx mori (Bm), Gryllus bimaculatus (Gb), Manduca sexta (Ms) and Tribolium castaenum (Tc). 

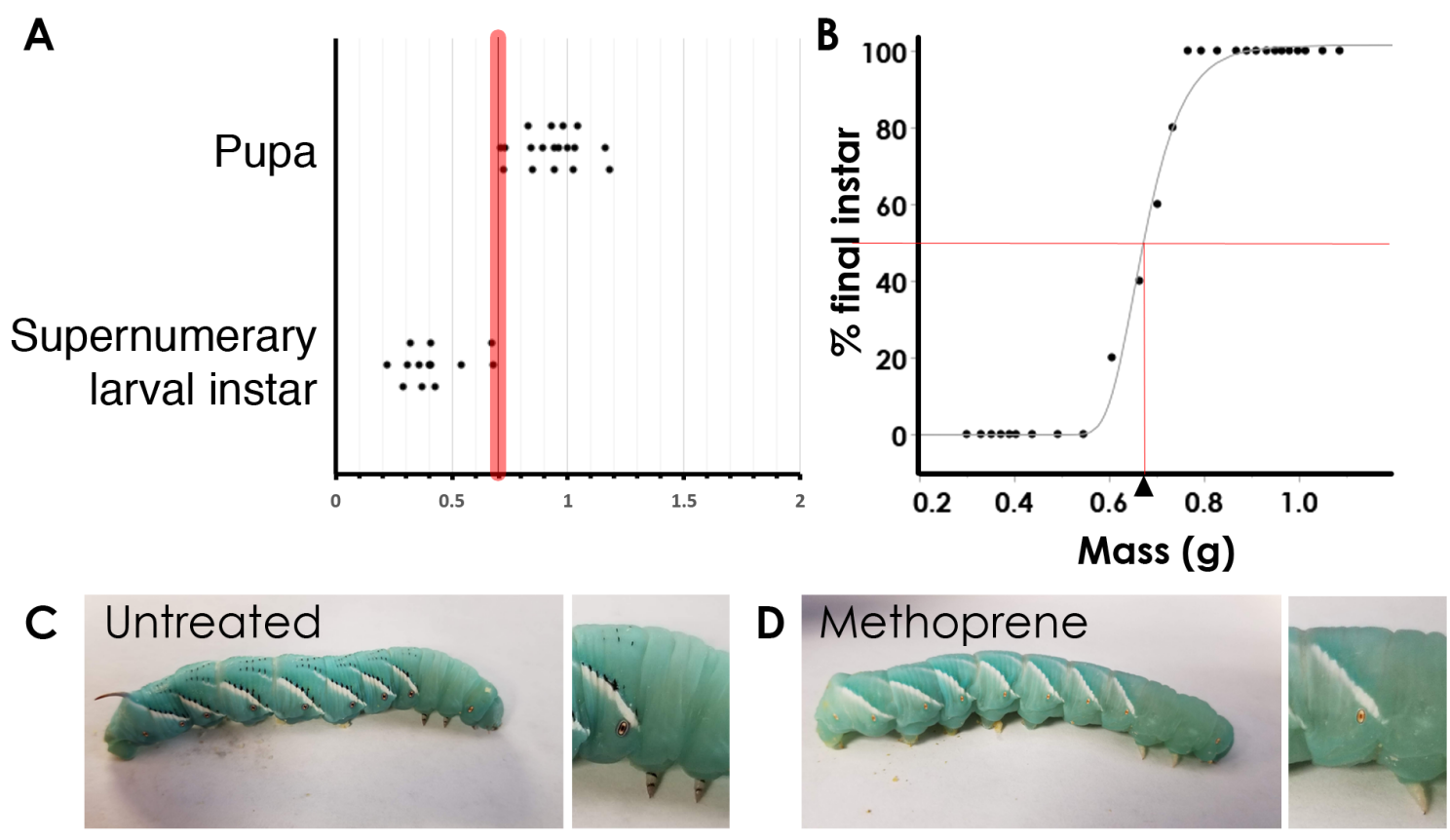

Supporting figure S6. Methoprene does not shift the threshold size. (A) Threshold size determination in methoprene-treated larvae that had undergone hypoxia treatment during the third instar. (B) The average masses of five larvae at the end of the fourth instar were plotted against the percentage of larvae that entered final larval instar. Triangle indicates the threshold size when $50 \%$ of the larvae wander at the end of the fifth instar. Line represents a Gompertz 3P model fit. (C) A normal fifth instar larva, showing the melaninic markings on the dorsal side and the legs. (D) A fifth instar that had been treated with methoprene. This larva weighed $1.18 \mathrm{~g}$ at 4 th HCS, well above the threshold size. Methoprene was applied on the dorsal side two days after 3rd instar HCS. 
Table S1: Ingredients for experimental diets used in this study. Percentages based on a standard (100\%) diet described by Yamamoto et al (1969)

\begin{tabular}{|l|r|r|}
\hline Ingredient & \multicolumn{1}{|c|}{$\begin{array}{c}\text { 100\% } \\
\text { diet }\end{array}$} & $\mathbf{4 0 \%}$ diet \\
\hline Gelcarin $(\mathrm{g})$ & 7.85 & 7.85 \\
\hline Distilled water $(\mathrm{mL})$ & 500 & 500 \\
\hline Wheat germ $(\mathrm{g})$ & 53.8 & 21.52 \\
\hline Casein $(\mathrm{g})$ & 24.2 & 9.68 \\
\hline Sucrose $(\mathrm{g})$ & 21.5 & 21.5 \\
\hline Torula yeast $(\mathrm{g})$ & 10.75 & 10.75 \\
\hline Cholesterol $(\mathrm{g})$ & 2.36 & 2.36 \\
\hline Wesson salt $(\mathrm{g})$ & 8.05 & 8.05 \\
\hline Sorbic acid $(\mathrm{g})$ & 1.35 & 1.35 \\
\hline Methyl paraben $(\mathrm{g})$ & 0.65 & 0.65 \\
\hline Ascorbic acid $(\mathrm{g})$ & 3.35 & 3.35 \\
\hline Streptomycin $(\mathrm{g})$ & 0.135 & 0.135 \\
\hline Kanamycin $(\mathrm{g})$ & 0.035 & 0.035 \\
\hline Formaldehyde $(\mathrm{mL})$ & 1.07 & 1.07 \\
\hline Linseed oil $(\mathrm{mL})$ & 0 & 0 \\
\hline Vitamin mixture $(\mathrm{mL})$ & 5 & 5 \\
\hline
\end{tabular}

Table S2: Primer sequences used in this study. *Sequences from Ono et al (2006). ** Sequences from Parthasarathy et al (2008).

\begin{tabular}{|l|l|l|l|}
\hline Gene & Purpose & Direction & Sequence \\
\hline \multirow{2}{*}{ MsMyo } & \multirow{2}{*}{ qPCR } & Forward & 5'-TACGCCTGGTTCGCTTGT-3' \\
\cline { 3 - 4 } & & Reverse & 5'-CTGCCACGGGAGAATTTAG-3' \\
\hline \multirow{2}{*}{ Msrpl17A* } & \multirow{2}{*}{ qPCR } & Forward & 5'-TCCGCATCTCACTGGGTCT-3' \\
\cline { 3 - 4 } & & Reverse & 5'-CACGGCAATCACATACAGGTT-3' \\
\hline \multirow{2}{*}{ Tcmyo } & \multirow{2}{*}{$\begin{array}{l}\text { KNAi } \\
\text { Knockdown } \\
\text { Tcrp49** }\end{array}$} & Forward & 5'-CCACGACATCCTCCACTTC-3' \\
\cline { 3 - 4 } & \multirow{2}{*}{$\begin{array}{l}\text { Knockdown } \\
\text { verification }\end{array}$} & Reverse & 5'-TACGGCTGGGTGACTTTCT-3' \\
\cline { 3 - 4 } & & Forward & 5'-GAGGAGGAGGACGACTACCA-3' \\
\cline { 3 - 4 } & & Reverse & 5'-TTCGGAGCGATGATGAAG-3' \\
\hline
\end{tabular}

\section{REFERENCES FOR SUPPORTING INFORMATION}

1. Yamamoto, R.T. (1969) Mass rearing of the tobacco hornworm. II. Larval rearing and pupation. J. Econ. Entomol. 62, 1427-1431.

2. Ono, H., Rewitz, K. F., Shinoda, T., Itoyama, K., Petryk, A., Rybczynski, R., et al. (2006) Spook and Spookier code for stage-specific components of the ecdysone biosynthetic pathway in Diptera. Dev Biol 298:555-570. 
bioRxiv preprint doi: https://doi.org/10.1101/2020.04.21.053835; this version posted April 23, 2020. The copyright holder for this preprint (which

was not certified by peer review) is the author/funder, who has granted bioRxiv a license to display the preprint in perpetuity. It is made available under aCC-BY-NC-ND 4.0 International license.

3. Parthasarathy, R., Tan, A., Bai, H., and Palli, S.R. (2008) Transcription factor broad suppresses precocious development of adult structures during larval-pupal metamorphosis in the red flour beetle, Tribolium castaneum. Mech. Develop. 125 , 299-313. 